\title{
3-D GPS velocity field and its implications on the present-day post-orogenic deformation of the Western Alps and Pyrenees
}

\author{
Hai Ninh Nguyen ${ }^{1}$, Philippe Vernant ${ }^{1}$, Stephane Mazzotti ${ }^{1}$, Giorgi Khazaradze ${ }^{2}$, and Eva Asensio ${ }^{2}$ \\ ${ }^{1}$ Laboratoire Géosciences Montpellier, Université Montpellier, Montpellier, France \\ ${ }^{2}$ Grup Risknat, Departament de Geodinàmica i Geofísica, Facultat de Geologia, Universitat de Barcelona (UB), \\ Martí i Franquès s/n, 08028 Barcelona, Spain
}

Correspondence to: Philippe Vernant (philippe.vernant@umontpellier.fr)

Received: 18 May 2016 - Published in Solid Earth Discuss.: 22 June 2016

Revised: 22 August 2016 - Accepted: 28 August 2016 - Published: 21 September 2016

\begin{abstract}
We present a new 3-D GPS velocity solution for 182 sites for the region encompassing the Western Alps, Pyrenees, and southern France. The velocity field is based on a Precise Point Positioning (PPP) solution, to which we apply a common-mode filter, defined by the 26 longest time series, in order to correct for network-wide biases (reference frame, unmodeled large-scale processes, etc.). We show that processing parameters, such as troposphere delay modeling, can lead to systematic velocity variations of $0.1-0.5 \mathrm{~mm} \mathrm{yr}^{-1}$ affecting both accuracy and precision, especially for short $(<5$ years) time series. A velocity convergence analysis shows that minimum time-series lengths of $\sim 3$ and $\sim 5.5$ years are required to reach a velocity stability of $0.5 \mathrm{~mm} \mathrm{yr}^{-1}$ in the horizontal and vertical components, respectively. On average, horizontal residual velocities show a stability of $\sim 0.2 \mathrm{~mm} \mathrm{yr}^{-1}$ in the Western Alps, Pyrenees, and southern France. The only significant horizontal strain rate signal is in the western Pyrenees with up to $4 \times 10^{-9} \mathrm{yr}^{-1} \mathrm{NNE}-\mathrm{SSW}$ extension, whereas no significant strain rates are detected in the Western Alps $\left(<1 \times 10^{-9} \mathrm{yr}^{-1}\right)$. In contrast, we identify significant uplift rates up to $2 \mathrm{~mm} \mathrm{yr}^{-1}$ in the Western Alps but not in the Pyrenees $\left(0.1 \pm 0.2 \mathrm{~mm} \mathrm{yr}^{-1}\right)$. A correlation between site elevations and fast uplift rates in the northern part of the Western Alps, in the region of the Würmian ice cap, suggests that part of this uplift is induced by postglacial rebound. The very slow uplift rates in the southern Western Alps and in the Pyrenees could be accounted for by erosion-induced rebound.
\end{abstract}

\section{Introduction}

The southwestern European and Mediterranean domains are part of the broad plate boundary zone that accommodates the relative motion between the Nubian and Eurasian plates. In this domain, active tectonics in the Western Alps and Pyrenees mountains are revealed by a moderate level of seismic activity, geological evidences for Quaternary deformation, and sparse geomorphological observations of recent deformation (e.g., Alasset and Meghraoui, 2005; Chardon et al., 2005; Chevrot et al., 2011; Lacan and Ortuño, 2012; Larroque et al., 2009). Global Positioning System (GPS) data show that central Europe east of the Rhine Graben and north of the Alps behaves rigidly at $\sim 0.4 \mathrm{~mm} \mathrm{yr}^{-1}$ and defines a stable European reference frame (e.g., Altamimi et al., 2011; Nocquet and Calais, 2003). To first order, horizontal deformation across the Alps and the Pyrenees is unresolvable at the level of GPS uncertainty, $\sim 0.5 \mathrm{~mm} \mathrm{yr}^{-1}$ (Nocquet, 2012; Rigo et al., 2015; Vigny et al., 2002), although recent studies suggest small possible extension in the French Alps (Walpersdorf et al., 2015) and the western Pyrenees (Asensio et al., 2012; Rigo et al., 2015). In contrast, studies of vertical velocities from GPS data indicate significant uplift rates in the Western and Central Alps (up to $\sim 2 \mathrm{~mm} \mathrm{yr}^{-1}$ ), decreasing toward the Eastern Alps (Serpelloni et al., 2013).

These very slow deformation rates in a stable plate context highlight the importance of characterization and quantification of GPS velocity uncertainties. Numerous sources of uncertainties affect GPS velocity estimations, especially in the vertical component: e.g., reference frame errors (Argus et al., 1999), seasonal signals (Blewitt and Lavallée, 2002), and 


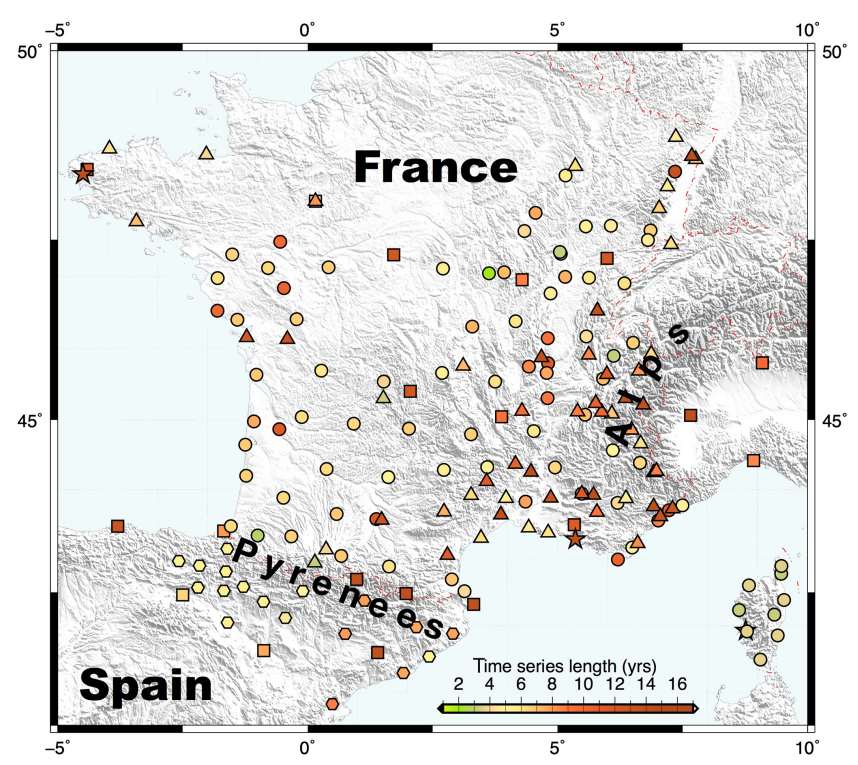

Figure 1. Distribution of the permanent stations used in this study. Symbols denote network (cf. text): stars - IGS; squares - EUREFEPN; triangles: RENAG; circles - RGP; hexagons: Spanish networks (Topo-Iberia, RGAN, CATNET, EPSH). Symbols are colorcoded according to time-series length.

atmospheric loading effects (Tregoning and Watson, 2009). These various sources of noise and non-tectonic signals can result in artificial trends over several years and systematic biases that may be correlated over large regions. Western Europe, as a near-stable region associated with low-deformation tectonic zones, provides an interesting test case to study GPS velocity and uncertainty estimations. Can tectonic velocities, likely below $1 \mathrm{~mm} \mathrm{yr}^{-1}$, be resolved for the Western Alps and the Pyrenees mountains? Can uplift patterns in these two mountain belts help constrain studies of ongoing tectonics and dynamics (e.g, Champagnac et al., 2007; Fox et al., 2015; Genti et al., 2015; Vernant et al., 2013)?

In this study, we analyze data from 182 permanent GPS stations located in France and Spain in order to characterize the horizontal and vertical deformation patterns in the Western Alps and Pyrenees. We focus on estimations of velocity uncertainties, mainly related to processing options and parameters, to define the minimum time span required to reach a set of horizontal and vertical velocity precision thresholds. These results are used to derive regional velocity and strain rate patterns in our study region.

\section{GPS data and processing}

We perform an analysis of GPS data from 1997 to December 2013 and provide a self-consistent velocity solution for 182 stations between longitudes -5 and $10^{\circ} \mathrm{E}$ and latitudes 41 and $49^{\circ} \mathrm{N}$, with time series from 1.9 to 16.0 years (average 7.8 years), cf. Fig. 1. Our analysis comprises stations from the following networks and operators: International GNSS Service (IGS, 3 stations), European Permanent GNSS (EUREF, 20 stations), Topo-Iberia (3 stations), Red de Geodesia Activa de Navarra (RGAN, 7 stations), Escuela Politécnica Superior de Huesca (EPSH, 1 station), La Xarxa CatNet de ICGC (6 stations), French Réseau National GPS (RENAG, 56 stations), and French Réseau GNSS Permanent (RGP, 86 stations); cf. Acknowledgement section for network and data references. Some of these networks include sites operated for geographic or cadastral purposes and are not always geodetic-quality monuments. We omit two sites of the RENAG network (BURE and JANU) because of numerous interruptions that cause significant nonlinearity to the time series.

For data processing, we use the Precise Point Positioning (PPP) software developed by the Canadian Geodetic Survey of Natural Resources Canada (CSRS) (Héroux and Kouba, 2001). This CSRS-PPP version 1.5 software provides GPS float solutions utilizing precise satellite orbits and clocks, satellite and receiver absolute antenna phase center (APC) mapping, tropospheric models (hydrostatic and wet delays, and mapping functions), solid Earth and ocean tide loading, and Earth rotation parameters. For our reference solution, we use IGS final products (Kouba, 2009) for satellite orbits and clocks, Earth rotation models, and phase mapping corrections for both satellite and ground-based antennas. We apply ocean-tide loading corrections based on the FES2004 model (Lyard et al., 2006). Tropospheric delays are estimated and corrected using Vienna mapping functions (VMF1, Boehm et al., 2006) with $10^{\circ}$ elevation cutoff angle, zenith delay estimated every $5 \mathrm{~min}$, and gradients every $6 \mathrm{~h}$. A priori troposphere parameters are derived from the European Centre for Medium-Range Weather Forecasts (http://www.ecmwf.int/).

We tested several processing options in order to estimate their impact on daily coordinates and estimated velocities. Comparisons with our reference solution show that the choice of tropospheric model has limited impact on final velocities. Using global mapping functions (GMF) instead of VMF1 results in mean differences of 0.2 and less than $0.1 \mathrm{~mm} \mathrm{yr}^{-1}$ in vertical and horizontal velocities, respectively. In contrast, the omission of Earth rotation models can result in significant impact in the PPP processing (Kouba, 2009). Our test solution without Earth rotation corrections yields horizontal and vertical velocities that vary from the reference solution by 0.3 and $0.5 \mathrm{~mm} \mathrm{yr}^{-1}$ on average. Similarly, the choice of APC mapping models can result in significant velocity variations. We illustrate this point by comparing a solution with the previous IGS model (igs05.atx, associated with IGS05 reference system; Rothacher and Schmid, 2006) with our reference solution that uses the current model (igs08.atx, associated with IGS08 reference system; Rebischung et al., 2011). North, east, and vertical velocities of these two solutions differ by $0.1,0.3$, and $0.5 \mathrm{~mm} \mathrm{yr}^{-1}$ on average. These tests indicate that the choice of processing parameters can lead to systematic velocity variations of the or- 

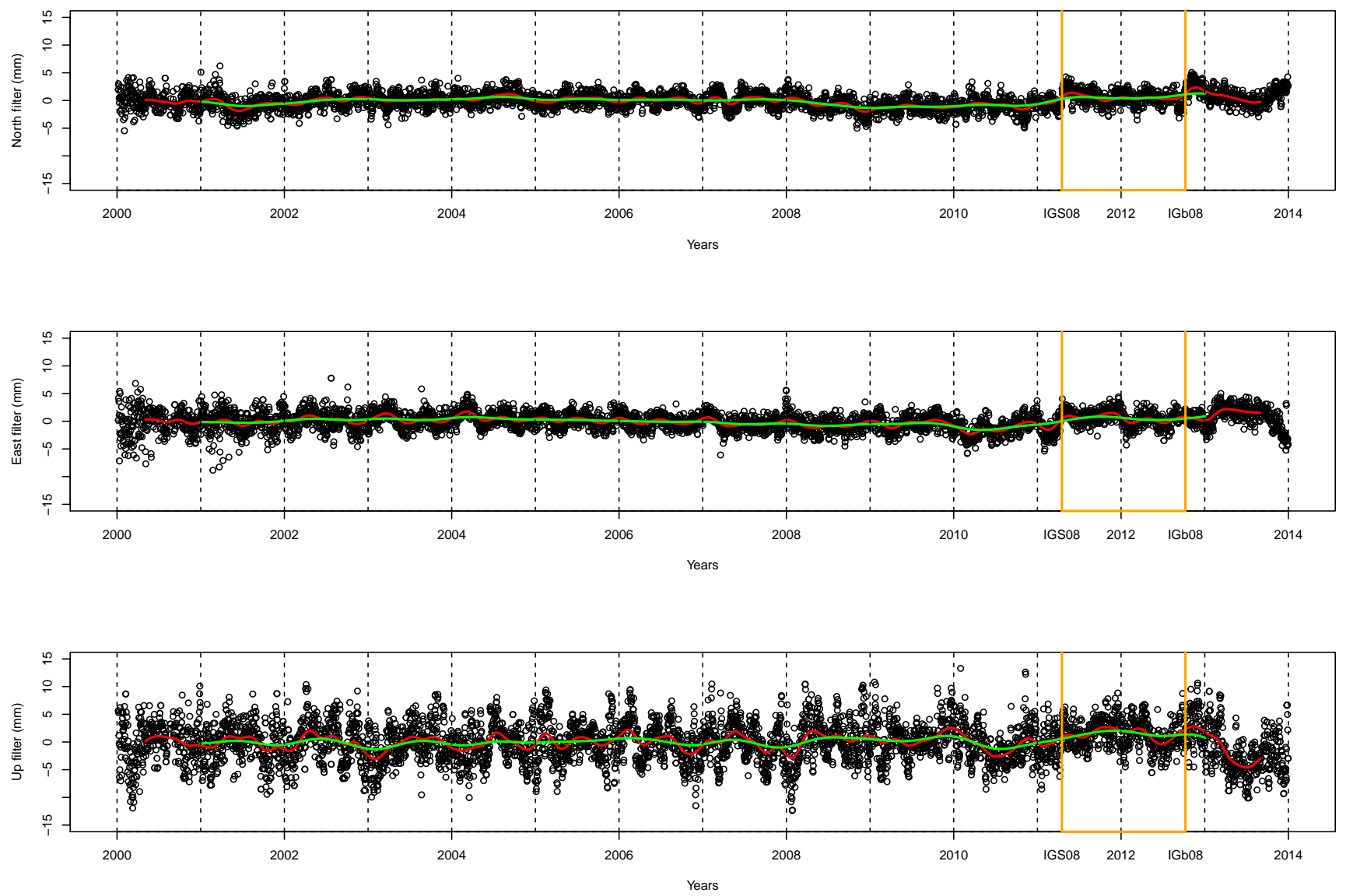

Figure 2. Common-mode stacked filter. Black circles show daily positions. Red and green curves show 1-month and 1-year time averages. Yellow vertical lines show the IGS05 - IGS08 and the IGS08 - IGb08 transitions.

der of $0.5 \mathrm{~mm} \mathrm{yr}^{-1}$, affecting primarily short ( $<5$ years) time series. Because the impacts of those parameter choices are dependent on the duration of the time series, the associated uncertainties affect both the velocity accuracies (e.g., whole network translations) and precisions (e.g., apparent relative motions between sites) and must be considered for tectonic analysis of GPS data, especially in extremely low-deforming areas such as western Europe.

\section{Time-series analysis}

\subsection{Time-series model}

Daily position time series are decomposed into a linear term (constant velocity), sine functions with annual and semiannual periods, and offsets defined by antenna, dome, and receiver changes as well as a visual inspection of the series. For each station's north, east, and vertical components, position time series are inverted using a least-square norm to find the best-fit amplitudes of all model parameters.

Uncertainties in GPS velocities are a function of the position repeatability, the length of the time series, the number of data points, the presence of steps in the time series, and the noise model (Agnew, 1992; Williams, 2003). For each station component, we perform a spectral analysis to estimate the spectral index and amplitude of the colored noise in the time series. We then use the formulation of Williams (2003) to calculate the standard error on the north, east, and vertical velocities. Spectral indices $\alpha$ for our analysis range between -0.4 and -1.1 , with a mean value $\alpha=-0.7$, indicating that noise in our PPP time series consists primarily of a combination of white $(\alpha=0)$ and flicker $(\alpha=-1)$ sources, with a very small random-walk $(\alpha=-2)$ contribution. Mean velocity standard errors are $0.5,0.4$, and $0.7 \mathrm{~mm} \mathrm{yr}^{-1}$ in the north, east, and vertical components, with a direct dependence on time-series length (cf. Sect. 4.1). This noise analysis is consistent with current estimations of noise type in GPS time series analyzed with PPP and double-difference software (e.g., Santamaría-Gómez et al., 2011).

\subsection{Reference frame}

Daily positions and velocities calculated from our PPP analysis are in a reference frame defined by the satellite orbits and clocks. For the time period of interest these frames fol- 
Table 1. Average offsets associated with the IGS reference frame transitions.

\begin{tabular}{lrrrr}
\hline & $\begin{array}{r}\text { Raw data } \\
\text { IGS05 - IGS08 }\end{array}$ & $\begin{array}{r}\text { Filtered data } \\
\text { IGS05 - IGS08 }\end{array}$ & $\begin{array}{r}\text { Raw data } \\
\text { IGS08 - Igb08 }\end{array}$ & $\begin{array}{r}\text { Filtered data } \\
\text { IGS08 - Igb08 }\end{array}$ \\
\hline North average (mm) & 2.4 & 0.4 & 1.9 & 0.9 \\
North standard deviation (mm) & 0.9 & 0.8 & 0.9 & 0.8 \\
East average (mm) & 2.0 & 0.3 & 1.5 & 0.6 \\
East standard deviation (mm) & 1.0 & 1.1 & 1.1 & 1.1 \\
Up average (mm) & 3.0 & -0.1 & -3.1 & -0.9 \\
Up standard deviation (mm) & 2.3 & 2.2 & 2.5 & 1.1 \\
\hline
\end{tabular}

Table 2. Residuals between our velocity solution and the ITRF2008 velocities (Altamimi et al., 2011).

\begin{tabular}{lrrr}
\hline Site & $\begin{array}{r}\text { East component } \\
\left(\mathrm{mm} \mathrm{yr}^{-1}\right)\end{array}$ & $\begin{array}{r}\text { North component } \\
\left(\mathrm{mm} \mathrm{yr}^{-1}\right)\end{array}$ & $\begin{array}{r}\text { Vertical component } \\
\left(\mathrm{mm} \mathrm{yr}^{-1}\right)\end{array}$ \\
\hline AJAC & 0.07 & 0.12 & -0.03 \\
BRST & 0.21 & -0.18 & -0.02 \\
CHIZ & -0.01 & 0.37 & 0.47 \\
GRAS & 0.02 & -0.14 & 0.25 \\
LROC & -0.09 & 0.08 & -0.28 \\
MARS & 0.06 & 0.04 & 0.66 \\
SJDV & -0.10 & 0.08 & 0.24 \\
TLSE & 0.08 & 0.17 & 0.05 \\
Mean $\left(\mathrm{mm} \mathrm{yr}^{-1}\right)$ & 0.07 & 0.03 & 0.17 \\
Standard deviation $\left(\mathrm{mm} \mathrm{yr}^{-1}\right)$ & 0.17 & 0.10 & 0.30 \\
\hline
\end{tabular}

low the official products of the "IGS Reprocessing 1" (e.g., Collilieux et al., 2009) and vary between IGS05 (up to 17 April 2011), IGS08 (between 17 April 2011 and 7 October 2012), and IGb08 (after 7 October 2012). The transformations between these three reference frames are not null, which results in potential offsets in our calculated positions. However, each processing group involved in the computation of the IGS products may not adopt synchronous model changes, resulting in variation in our time series that cannot be corrected by a single break (T. Herring, personal communication, 2015).

In order to correct for these possible biases, we adopt an empirical approach that consists in removing residual signals common to the whole network. We first calculate a commonmode filter for each component by stacking the daily residuals of the time series (post time-series model, cf. Sect. 3.1) for a subset of the 26 stations with 10 years or more of data, $80 \%$ of data completeness, and a network-wide coverage. This filter is then subtracted from the original time series of all 182 stations to produce filtered series that are re-analyzed. Figure 2 shows the common-mode filter. We perform a statistical analysis to estimate the effectiveness of this method by estimating the amplitude of offsets in the time series forced at the IGS05 - IGS08 and IGS08 - Igb08 transition dates. For the original (non-filtered) data, significant offsets are estimated in all three components (about $1.5-3 \mathrm{~mm}$ on average, Table 1). In contrast, the filtered data result in much smaller offsets $(0.1-0.9 \mathrm{~mm}$, Table 1$)$ that are not significant compared to their standard deviation. Thus, we consider that our filtered solution is essentially free from reference frame variation problems. This analysis also highlights the importance of the new "Reprocessing 2" analysis currently on going within the IGS.

Velocities calculated after the common-mode filter procedure are aligned with a "network average" reference frame, which is not directly aligned with a specific International Terrestrial Reference Frame (ITRF) realization. Our analysis includes eight stations of the core IGS network for which official positions and velocities are provided in the latest ITRF2008 (Altamimi et al., 2011). Time series for these stations range between 11 and 16 year-lengths, resulting in well-constrained velocities. A comparison of our velocities with those from ITRF2008 is given in Table 2. The mean differences in the north, east, and vertical components are within their standard errors $(0.03 \pm 0.10,0.07 \pm 0.17$, $\left.0.17 \pm 0.30 \mathrm{~mm} \mathrm{yr}^{-1}\right)$, indicating no significant difference between our solution and ITRF2008 at the level of $\sim 0.1$ and $\sim 0.3 \mathrm{~mm} \mathrm{yr}^{-1}$ in the horizontal and vertical components, respectively.

\section{Precisions and uncertainties on velocities}

In order to assess the impact of time-series length on the estimated velocity for our PPP solution, we perform a conver- 


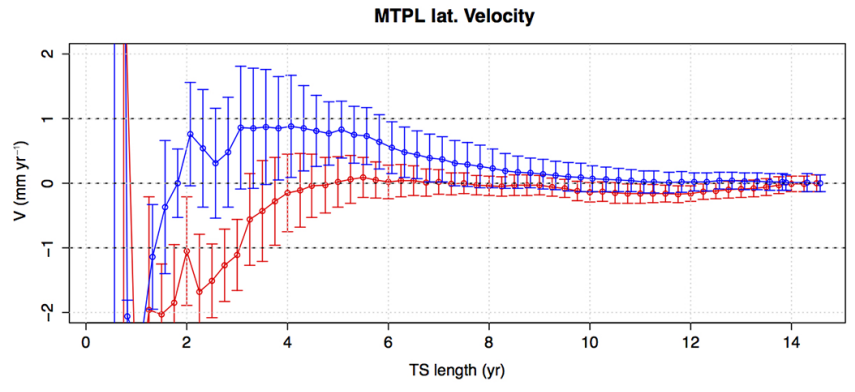

$\sim 5$ and $\sim 3$ years for the original and filtered data, respectively. Similarly, the convergence time decreases from $\sim 6.5$ to $\sim 5.5$ years in the vertical component. Convergence times estimated for a $0.1 \mathrm{~mm} \mathrm{yr}^{-1}$ velocity threshold are probably a minimum as about half of the time series used for this estimation have a length approaching the convergence time.

Kierulf et al. (2013) performed a similar convergence analysis for $\sim 140$ GPS stations in Norway. They estimate convergence times of 3.0, 2.5, and 3.5 years for a velocity convergence threshold of $0.5 \mathrm{~mm} \mathrm{yr}^{-1}$ in the north, east, and vertical components, respectively. These estimations are similar to ours for the horizontal components and shorter by about 2 years for the vertical. This shorter convergence times may be due to (1) a rougher time step (6 months vs. 1 month) that limits their time resolution and (2) inclusion of short $(<10$ years) time series in their statistics, which biases the convergence time estimations to shorter values. The effect of double-difference processing, intrinsically less noisy than our PPP solution, may also contribute to shorter convergence times.

Because it removes a significant long-period noise component from the time series, the common-mode filter also has a strong impact on the estimation of velocity standard errors. The velocity uncertainty analysis (Sect. 3.1) performed on filtered data yields spectral indices significantly smaller (mean value $\alpha=-0.45$ vs. $\alpha=-0.7$ for the original data) and thus whiter spectra. This effect is illustrated in Fig. 5 on the spectra for station MTPL original and filtered data. A direct consequence of these whiter spectra is the reduction by a factor of $\sim 2$ of the mean north, east, and vertical velocity standard errors compared to the original data (i.e., 0.2, 0.2, and $0.3 \mathrm{~mm} \mathrm{yr}^{-1}$ vs. $0.5,0.4$, and $0.7 \mathrm{~mm} \mathrm{yr}^{-1}$ for the unfiltered data).

gence analysis for each site in which we compare the velocity computed using the full time-series length to velocities computed for a range of time-series lengths of 1 year and more. We estimate the minimum time required for the time-varying velocities to be similar to the long-term velocities within four thresholds values $\left(1.0,0.5,0.2\right.$, and $\left.0.1 \mathrm{~mm} \mathrm{yr}^{-1}\right)$. This analysis is performed by extending the analyzed data by time steps of 2 months, starting from both the beginning (forward) and end (backward) of the series. Figure 3 presents an example for station MTPL of velocity evolution as a function of the time-series length relative to the reference (full length) velocity. For this station, the short-term velocities can deviate from the full-length reference by $1 \mathrm{~mm} \mathrm{yr}^{-1}$ or more for series shorter than 3-4 years.

The results of this analysis for the 44 stations with data longer than 10 years are summarized in Fig. 4. Convergence times for the filtered data are systematically shorter (by about 1-2 years) compared to the original data due to the removal of long-period non-tectonic signals. For example, the minimum time-series lengths required to reach a stability threshold of $0.5 \mathrm{~mm} \mathrm{yr}^{-1}$ in the horizontal components are

\section{Velocity solution}

On the basis of the analyses described above, we present a final GPS velocity field using the filtered data (Table 3). The velocities for 139 stations with time series longer than 5 years, which correspond to an average velocity precision (convergence threshold) of $\sim 0.5 \mathrm{~mm} \mathrm{yr}^{-1}$ or better and $1 \sigma$ horizontal velocity uncertainties smaller than $0.5 \mathrm{~mm} \mathrm{yr}^{-1}$, are represented in Fig. 6. We define a regional reference frame by minimizing, through the estimation of an Euler vector, the velocities of 61 sites over the whole network and with time series longer than 6 years (see Table 3 ). The mean values of the residual velocities are 0.1 and $0.06 \mathrm{~mm} \mathrm{yr}^{-1}$ for the north and east component, respectively. This result is in agreement with previous studies (e.g., Altamimi et al., 2011; Argus et al., 2010; Nocquet and Calais, 2003; Nocquet, 2012) and shows that horizontal deformation is very slow, including in the Western Alps and Pyrenees. The only site with a large velocity $\left(>2 \mathrm{~mm} \mathrm{yr}^{-1}\right)$ corresponds to a station of the foot of a landslide (CLAP, southwestern Alps). 
Table 3. Site locations and velocities in a regional (western Europe) reference frame as determined in this study by minimizing the velocities of the 61 sites indicated by an asterisk. East, north, and vertical GPS velocity components $\left(V_{\mathrm{E}}, V_{\mathrm{N}}, V_{\mathrm{Up}}\right)$ and $1 \sigma$ uncertainties $\left(\sigma_{\mathrm{E}}, \sigma_{\mathrm{N}}, \sigma_{\mathrm{Up}}\right)$ are given in $\mathrm{mm} \mathrm{yr}^{-1}$. "Dur." corresponds to the length of the time series.

\begin{tabular}{|c|c|c|c|c|c|c|c|c|c|c|}
\hline $\begin{array}{l}\text { Site } \\
\text { name }\end{array}$ & $\begin{array}{l}\text { Long. } \\
\left({ }^{\circ} \mathrm{E}\right)\end{array}$ & $\begin{array}{l}\text { Lat. } \\
\left({ }^{\circ} \mathrm{N}\right)\end{array}$ & $\begin{array}{l}\text { Alt. } \\
\text { (m) }\end{array}$ & $\begin{array}{r}V_{\mathrm{E}} \\
\left(\mathrm{mm} \mathrm{yr}^{-1}\right)\end{array}$ & $\begin{array}{r}V_{\mathrm{N}} \\
\left(\mathrm{mm} \mathrm{yr}^{-1}\right)\end{array}$ & $\begin{array}{r}V_{\mathrm{Up}} \\
\left(\mathrm{mm} \mathrm{yr}^{-1}\right)\end{array}$ & $\begin{array}{r}\sigma_{\mathrm{E}} \\
\left(\mathrm{mm} \mathrm{yr}^{-1}\right)\end{array}$ & $\begin{array}{r}\sigma_{\mathrm{N}} \\
\left(\mathrm{mm} \mathrm{yr}^{-1}\right)\end{array}$ & $\begin{array}{r}\sigma_{\mathrm{Up}} \\
\left(\mathrm{mm} \mathrm{yr}^{-1}\right)\end{array}$ & $\begin{array}{r}\text { Dur. } \\
(\mathrm{yr})\end{array}$ \\
\hline AGDE* & 3.4664 & 43.2964 & 65.8 & -0.21 & -0.01 & -0.50 & 0.28 & 0.46 & 0.18 & 6.29 \\
\hline AICI & -1.0014 & 43.3356 & 121.4 & 0.69 & 0.09 & -0.70 & 0.45 & 0.58 & 0.44 & 2.86 \\
\hline AIGL* & 3.5813 & 44.1214 & 1618.8 & 0.28 & -0.22 & 0.98 & 0.07 & 0.13 & 0.25 & 11.31 \\
\hline AJAC* & 8.7626 & 41.9275 & 98.8 & -0.12 & 0.15 & 0.29 & 0.05 & 0.03 & 0.12 & 13.94 \\
\hline ALPE & 6.0835 & 45.0866 & 1892.3 & -0.32 & 0.04 & 1.06 & 0.07 & 0.05 & 0.21 & 6.81 \\
\hline ALSA & -2.1640 & 42.8920 & 584.2 & -0.70 & 0.90 & 0.03 & 0.10 & 0.08 & 0.23 & 5.35 \\
\hline ALUY & 3.6268 & 47.0419 & 324.1 & -0.04 & 1.13 & -0.27 & 0.07 & 0.12 & 0.47 & 1.94 \\
\hline AMB2 & 3.7501 & 45.5406 & 617.6 & 0.42 & 0.07 & -0.77 & 0.10 & 0.05 & 0.25 & 6.16 \\
\hline ANCY & 6.1233 & 45.9007 & 528.8 & 0.39 & 0.71 & -1.00 & 0.23 & 0.08 & 0.19 & 3.16 \\
\hline ANGE* & -0.5479 & 47.4719 & 106.1 & -0.27 & -0.03 & -0.06 & 0.06 & 0.04 & 0.37 & 9.90 \\
\hline ANGL* & -1.4061 & 46.4056 & 56.7 & 0.38 & -0.24 & 0.00 & 0.10 & 0.06 & 0.15 & 6.78 \\
\hline ASIN & -0.0990 & 42.5170 & 1694.9 & 0.17 & 0.29 & 1.81 & 0.11 & 0.06 & 0.58 & 4.99 \\
\hline ATST & 9.3360 & 42.1683 & 740.4 & -0.53 & 0.60 & -1.81 & 0.11 & 0.05 & 0.33 & 3.18 \\
\hline AUBU & 7.1967 & 48.2169 & 1151.9 & 0.36 & -0.01 & -0.71 & 0.17 & 0.24 & 0.79 & 5.49 \\
\hline $\mathrm{AUCH}$ & 0.5806 & 43.6495 & 232.0 & 0.67 & -0.08 & -0.79 & 0.10 & 0.04 & 0.33 & 6.78 \\
\hline AUTN* & 4.2890 & 46.9538 & 353.6 & 0.10 & -0.04 & -0.89 & 0.07 & 0.03 & 0.24 & 8.71 \\
\hline AVEL & 0.7510 & 41.8820 & 682.7 & 0.22 & -2.02 & 3.11 & 0.63 & 0.25 & 0.62 & 7.89 \\
\hline AXPV* & 5.3332 & 43.4912 & 229.4 & -0.15 & 0.25 & -0.25 & 0.14 & 0.06 & 0.33 & 11.27 \\
\hline BACT & 6.6496 & 44.3877 & 1205.1 & -0.15 & -0.67 & -0.23 & 0.06 & 0.09 & 0.26 & 6.39 \\
\hline BANN & 4.1563 & 44.3692 & 357.6 & 0.04 & 0.00 & 0.40 & 0.04 & 0.04 & 0.45 & 10.49 \\
\hline BARY & 0.6719 & 43.0357 & 633.5 & 0.63 & 0.21 & -0.39 & 0.16 & 0.05 & 0.15 & 6.77 \\
\hline BAUB & 3.9670 & 43.8769 & 210.9 & 0.34 & 0.03 & 0.43 & 0.09 & 0.06 & 0.38 & 5.43 \\
\hline BEA2 & 3.1367 & 42.5153 & 108.2 & 1.34 & 0.05 & -0.35 & 0.12 & 0.48 & 0.16 & 4.14 \\
\hline BELL* & 1.4000 & 41.6000 & 53.4 & -0.27 & -0.08 & 0.81 & 0.11 & 0.08 & 0.15 & 13.98 \\
\hline BIAZ & -1.5369 & 43.4720 & 121.4 & 0.83 & 0.31 & -0.75 & 0.06 & 0.07 & 0.19 & 6.35 \\
\hline BLFT & 6.8585 & 47.6259 & 416.5 & -0.35 & 0.04 & 0.16 & 0.08 & 0.06 & 0.45 & 6.63 \\
\hline BLGN & 5.5741 & 46.1718 & 544.7 & -0.04 & 0.55 & -3.40 & 0.18 & 0.04 & 0.43 & 4.26 \\
\hline BLIX & 6.3667 & 43.8735 & 1077.2 & 0.22 & 0.29 & -0.32 & 0.06 & 0.07 & 0.13 & 4.68 \\
\hline BRDO & 9.4749 & 42.7746 & 69.5 & -0.12 & 0.49 & -1.89 & 0.04 & 0.13 & 0.77 & 3.14 \\
\hline BRES* & -0.4735 & 46.8397 & 245.2 & 0.13 & 0.00 & -0.07 & 0.07 & 0.04 & 0.18 & 10.24 \\
\hline BRST & -4.4966 & 48.3805 & 65.8 & -0.40 & 0.31 & 0.11 & 0.05 & 0.06 & 0.06 & 13.98 \\
\hline $\mathrm{BSCN}^{*}$ & 5.9894 & 47.2469 & 359.5 & -0.07 & -0.06 & 0.39 & 0.08 & 0.02 & 0.07 & 12.21 \\
\hline BUAN & 5.3536 & 48.4862 & 416.3 & 0.05 & 0.28 & 0.47 & 0.23 & 0.09 & 0.29 & 6.13 \\
\hline CACI* & 3.9328 & 47.0569 & 610.2 & -0.36 & 0.05 & -0.63 & 0.10 & 0.02 & 0.35 & 7.06 \\
\hline CAMP & 8.7908 & 41.9182 & 60.8 & 0.38 & 0.66 & -0.84 & 0.13 & 0.08 & 0.52 & 3.63 \\
\hline CANT & -3.8000 & 43.4700 & 99.0 & -0.37 & 0.51 & 0.13 & 0.06 & 0.04 & 0.07 & 13.00 \\
\hline CARQ* & -1.5091 & 47.2990 & 88.1 & 0.08 & -0.10 & 0.07 & 0.16 & 0.07 & 0.24 & 6.52 \\
\hline CASS & 2.9040 & 41.8830 & 251.8 & -0.10 & 0.49 & -0.17 & 0.24 & 0.07 & 0.20 & 7.74 \\
\hline CAUS & 1.6150 & 44.1816 & 227.0 & -0.87 & 0.32 & -2.62 & 0.38 & 0.05 & 1.20 & 5.00 \\
\hline CBRY & 5.9092 & 45.5813 & 324.7 & 0.00 & 0.13 & -0.54 & 0.09 & 0.03 & 0.18 & 7.16 \\
\hline CHAL & 4.8587 & 46.7673 & 234.1 & 0.62 & 0.03 & -2.24 & 0.25 & 0.12 & 0.61 & 4.71 \\
\hline CHAM* & 5.8811 & 45.1107 & 1874.6 & -0.10 & -0.09 & 0.97 & 0.06 & 0.04 & 0.18 & 10.03 \\
\hline CHAS* & 4.5594 & 47.8627 & 299.4 & -0.08 & -0.08 & 0.06 & 0.15 & 0.03 & 0.21 & 7.52 \\
\hline CHIZ* & -0.4077 & 46.1335 & 113.2 & 0.05 & -0.16 & 0.18 & 0.03 & 0.03 & 0.03 & 13.19 \\
\hline CHMX & 6.8730 & 45.9262 & 1120.8 & -1.50 & -0.60 & 0.50 & 0.29 & 0.59 & 0.36 & 6.33 \\
\hline CHRN* & 4.8617 & 43.8814 & 126.8 & -0.04 & 0.03 & 0.04 & 0.03 & 0.02 & 0.08 & 13.17 \\
\hline CHTL & 6.3586 & 45.3041 & 850.3 & -0.67 & -0.04 & 2.46 & 0.04 & 0.05 & 0.13 & 13.98 \\
\hline CLAP & 6.9271 & 44.2484 & 1370.0 & 2.02 & 1.65 & -1.67 & 0.23 & 0.12 & 0.41 & 8.91 \\
\hline CLFD & 3.1111 & 45.7610 & 473.6 & 0.35 & 0.13 & 0.18 & 0.12 & 0.04 & 0.08 & 7.15 \\
\hline CNNS & 7.0157 & 43.5546 & 88.0 & 0.34 & 0.28 & -0.38 & 0.04 & 0.05 & 0.32 & 8.59 \\
\hline COMO* & 9.0956 & 45.8022 & 292.3 & -0.15 & 0.04 & -0.21 & 0.06 & 0.06 & 0.12 & 9.78 \\
\hline COUT & -0.1176 & 45.0410 & 81.8 & 0.26 & 0.16 & -0.34 & 0.07 & 0.07 & 0.23 & 6.28 \\
\hline CRAL & 0.3672 & 43.1284 & 658.4 & 0.30 & 0.73 & -0.34 & 0.12 & 0.07 & 0.23 & 3.89 \\
\hline CREU & 3.3200 & 42.3200 & 133.4 & 0.32 & -0.01 & -0.18 & 0.28 & 0.04 & 0.30 & 13.98 \\
\hline CUBX & -0.5664 & 44.8689 & 59.0 & -0.33 & -0.26 & -0.91 & 0.06 & 0.08 & 0.19 & 11.24 \\
\hline
\end{tabular}


Table 3. Continued.

\begin{tabular}{|c|c|c|c|c|c|c|c|c|c|c|}
\hline $\begin{array}{l}\text { Site } \\
\text { name }\end{array}$ & $\begin{array}{l}\text { Long. } \\
\left({ }^{\circ} \mathrm{E}\right)\end{array}$ & $\begin{array}{l}\text { Lat. } \\
\left({ }^{\circ} \mathrm{N}\right)\end{array}$ & $\begin{array}{l}\text { Alt. } \\
\text { (m) }\end{array}$ & $\begin{array}{r}V_{\mathrm{E}} \\
\left(\mathrm{mm} \mathrm{yr}^{-1}\right)\end{array}$ & $\begin{array}{r}V_{\mathrm{N}} \\
\left(\mathrm{mm} \mathrm{yr}^{-1}\right)\end{array}$ & $\begin{array}{r}V_{\mathrm{Up}} \\
\left(\mathrm{mm} \mathrm{yr}^{-1}\right)\end{array}$ & $\begin{array}{r}\sigma_{\mathrm{E}} \\
\left(\mathrm{mm} \mathrm{yr}^{-1}\right)\end{array}$ & $\begin{array}{r}\sigma_{\mathrm{N}} \\
\left(\mathrm{mm} \mathrm{yr}^{-1}\right)\end{array}$ & $\begin{array}{r}\sigma_{\mathrm{Up}} \\
\left(\mathrm{mm} \mathrm{yr}^{-1}\right)\end{array}$ & $\begin{array}{r}\text { Dur. } \\
\text { (yr) }\end{array}$ \\
\hline DIJO & 5.0651 & 47.3106 & 328.7 & 0.51 & 0.09 & -0.34 & 0.13 & 0.10 & 0.31 & 4.71 \\
\hline DOJX & 5.1605 & 48.3629 & 287.9 & -0.57 & -0.31 & -0.41 & 0.21 & 0.80 & 0.36 & 5.43 \\
\hline EBRE & 0.4920 & 40.8210 & 107.8 & 0.32 & -0.50 & 0.06 & 0.08 & 0.07 & 0.08 & 8.72 \\
\hline EGLT & 2.0520 & 45.4034 & 667.0 & 0.06 & -0.49 & 0.69 & 0.07 & 0.08 & 0.20 & 12.21 \\
\hline EOST & 7.7625 & 48.5798 & 213.4 & 0.41 & -0.13 & 0.72 & 0.20 & 0.21 & 0.40 & 6.62 \\
\hline EPSH & -0.4480 & 42.1190 & 522.8 & -1.36 & -1.40 & 4.42 & 0.14 & 0.22 & 1.44 & 4.56 \\
\hline ERCK & 7.3642 & 48.8730 & 296.2 & -0.14 & 0.11 & 0.15 & 0.07 & 0.06 & 0.44 & 4.66 \\
\hline ESAB* & 4.7979 & 45.3071 & 207.7 & 0.11 & 0.00 & -0.78 & 0.38 & 0.09 & 0.31 & 8.87 \\
\hline ESCO* & 0.9800 & 42.6900 & 2508.4 & 0.06 & 0.06 & 0.07 & 0.06 & 0.03 & 0.09 & 13.98 \\
\hline EZEV & 7.4973 & 43.7744 & 76.7 & -0.15 & 0.46 & -0.45 & 0.10 & 0.09 & 0.48 & 4.84 \\
\hline FCLZ* & 5.9857 & 45.6432 & 1374.2 & -0.02 & 0.11 & 0.95 & 0.16 & 0.06 & 0.42 & 12.00 \\
\hline FERR & -1.2488 & 44.6460 & 106.2 & 0.62 & -0.01 & -3.85 & 0.92 & 0.48 & 1.50 & 6.72 \\
\hline FJCP & 2.7949 & 43.0482 & 322.7 & -0.44 & -0.02 & 0.80 & 0.09 & 0.03 & 0.14 & 11.19 \\
\hline FLRC & 3.5945 & 44.3253 & 607.9 & -1.45 & -0.26 & -0.32 & 0.15 & 0.11 & 0.19 & 4.86 \\
\hline FRTT & 5.5663 & 47.6803 & 373.5 & -0.10 & -0.06 & -1.06 & 5.56 & 0.30 & 2.04 & 5.43 \\
\hline FUEN & -0.8850 & 42.3600 & 924.8 & 0.28 & 0.03 & -0.19 & 0.08 & 0.04 & 0.15 & 5.15 \\
\hline GARR & 1.9140 & 41.2930 & 634.5 & -0.02 & 0.16 & -0.92 & 0.11 & 0.04 & 0.77 & 7.72 \\
\hline GDIJ & 5.0447 & 47.3327 & 315.2 & 0.58 & 0.53 & -0.24 & 0.32 & 0.21 & 0.28 & 3.19 \\
\hline GENO* & 8.9211 & 44.4194 & 152.7 & -0.19 & -0.13 & 0.06 & 0.05 & 0.02 & 0.17 & 8.43 \\
\hline GINA & 5.7870 & 43.6757 & 382.0 & -0.13 & -0.15 & 0.16 & 0.03 & 0.03 & 0.06 & 9.02 \\
\hline GLRA & 4.5241 & 44.8393 & 813.9 & -0.84 & 0.10 & -1.62 & 0.05 & 0.03 & 0.27 & 5.86 \\
\hline GRAS* & 6.9206 & 43.7547 & 1319.3 & 0.08 & 0.22 & 0.15 & 0.07 & 0.03 & 0.07 & 13.98 \\
\hline GRJF & 1.5139 & 45.3029 & 458.6 & 0.43 & 0.40 & 0.18 & 0.14 & 0.08 & 0.72 & 3.27 \\
\hline GRON & 2.7082 & 47.1067 & 222.8 & 0.61 & 0.37 & -0.48 & 0.24 & 0.11 & 0.25 & 5.53 \\
\hline GUIL & 6.6619 & 44.6622 & 1171.2 & -0.33 & 0.35 & 0.80 & 0.08 & 0.24 & 0.45 & 6.13 \\
\hline GUIP* & -4.4118 & 48.4446 & 154.7 & 0.22 & -0.05 & -0.45 & 0.05 & 0.03 & 0.14 & 11.20 \\
\hline ILBO* & 0.4179 & 47.1246 & 90.5 & 0.41 & 0.14 & -0.35 & 0.05 & 0.03 & 0.40 & 6.63 \\
\hline JOUX* & 5.7958 & 46.5287 & 878.9 & -0.33 & 0.13 & -0.12 & 0.08 & 0.03 & 0.17 & 13.70 \\
\hline LACA & 2.7276 & 43.6810 & 1326.3 & -0.03 & 0.27 & 0.06 & 0.06 & 0.06 & 0.16 & 7.48 \\
\hline LBUG & 0.9211 & 44.9454 & 266.5 & 0.41 & 0.45 & -0.78 & 0.27 & 0.07 & 0.33 & 6.35 \\
\hline LCAU* & -1.0755 & 44.9782 & 73.7 & 0.37 & 0.27 & -0.50 & 0.06 & 0.05 & 0.14 & 7.52 \\
\hline LEBE* & 5.6246 & 45.9161 & 940.5 & 0.11 & 0.14 & 0.71 & 0.05 & 0.05 & 0.12 & 8.45 \\
\hline LFAZ* & 5.3990 & 45.1166 & 1014.0 & -0.11 & 0.00 & 1.03 & 0.05 & 0.03 & 0.23 & 8.78 \\
\hline LGAR* & 0.3789 & 44.2974 & 136.1 & 0.17 & 0.13 & -0.34 & 0.09 & 0.07 & 0.17 & 6.30 \\
\hline LLIV* & 1.9700 & 42.4800 & 1466.8 & 0.12 & -0.10 & 0.18 & 0.02 & 0.03 & 0.04 & 13.98 \\
\hline LNDA & -2.5780 & 42.9600 & 804.4 & 0.32 & 0.13 & 0.59 & 0.11 & 0.11 & 0.26 & 5.10 \\
\hline LOSA & -2.1950 & 42.5680 & 510.3 & 0.27 & 0.05 & 0.16 & 0.12 & 0.10 & 0.19 & 5.36 \\
\hline LROC* & -1.2193 & 46.1589 & 57.9 & 0.04 & -0.13 & -0.02 & 0.03 & 0.02 & 0.04 & 12.07 \\
\hline LUCE & 7.2682 & 47.4384 & 741.6 & -0.13 & 0.53 & -0.19 & 0.04 & 0.07 & 0.51 & 6.16 \\
\hline LUCI & 9.5309 & 42.3863 & 63.7 & 0.54 & 0.55 & -0.26 & 0.15 & 0.13 & 0.13 & 3.63 \\
\hline LUMI & 8.8274 & 42.6029 & 57.0 & 0.18 & 0.94 & -0.76 & 0.29 & 0.07 & 0.33 & 3.63 \\
\hline LURI & 9.4759 & 42.8884 & 54.2 & 0.15 & 1.26 & -0.71 & 0.28 & 0.08 & 0.58 & 3.54 \\
\hline $\mathrm{MACH}$ & -1.8029 & 46.9782 & 64.6 & 0.78 & 0.39 & -0.51 & 0.06 & 0.05 & 0.11 & 6.16 \\
\hline MAKS & 7.0315 & 47.9230 & 1237.2 & 0.14 & 0.14 & 0.50 & 0.15 & 0.10 & 0.78 & 6.64 \\
\hline MAN2 & 0.1553 & 48.0186 & 168.0 & 0.26 & 0.16 & -0.34 & 0.14 & 0.09 & 0.13 & 5.80 \\
\hline MANS* & 0.1553 & 48.0186 & 168.0 & -0.26 & 0.20 & 0.62 & 0.11 & 0.11 & 0.45 & 8.13 \\
\hline MARG & 6.5107 & 46.0844 & 524.2 & -0.20 & 0.43 & -0.63 & 0.11 & 0.06 & 0.28 & 6.63 \\
\hline MARS & 5.3538 & 43.2788 & 61.8 & -0.29 & -0.25 & -0.21 & 0.03 & 0.01 & 0.09 & 13.98 \\
\hline MATA & 2.4290 & 41.5400 & 123.6 & -0.80 & 0.15 & 0.72 & 0.07 & 0.03 & 0.18 & 5.14 \\
\hline MDOR* & 4.8090 & 45.7990 & 330.6 & -0.10 & -0.12 & -0.33 & 0.04 & 0.03 & 0.13 & 11.21 \\
\hline $\mathrm{MICH}^{*}$ & 5.7174 & 43.9242 & 628.2 & -0.14 & 0.02 & 0.26 & 0.04 & 0.05 & 0.05 & 13.98 \\
\hline MIMZ & -1.2283 & 44.2006 & 71.7 & 0.83 & 0.31 & 0.07 & 0.04 & 0.05 & 0.11 & 6.63 \\
\hline MNBL & 6.8061 & 47.4958 & 413.1 & -0.29 & -0.08 & -0.43 & 0.27 & 0.09 & 0.39 & 4.71 \\
\hline MODA* & 6.7101 & 45.2138 & 1182.3 & -0.11 & -0.23 & 1.25 & 0.03 & 0.02 & 0.42 & 13.98 \\
\hline MOGN & 4.8029 & 46.1483 & 233.0 & 0.39 & 0.20 & -1.74 & 0.09 & 0.06 & 0.15 & 9.19 \\
\hline MONB & 4.3381 & 47.6190 & 303.9 & 0.46 & -0.07 & -0.42 & 0.86 & 0.63 & 0.87 & 4.10 \\
\hline
\end{tabular}


Table 3. Continued.

\begin{tabular}{|c|c|c|c|c|c|c|c|c|c|c|}
\hline $\begin{array}{l}\text { Site } \\
\text { name }\end{array}$ & $\begin{array}{l}\text { Long. } \\
\left({ }^{\circ} \mathrm{E}\right)\end{array}$ & $\begin{array}{l}\text { Lat. } \\
\left({ }^{\circ} \mathrm{N}\right)\end{array}$ & $\begin{array}{l}\text { Alt. } \\
(\mathrm{m})\end{array}$ & $\begin{array}{r}V_{\mathrm{E}} \\
\left(\mathrm{mm} \mathrm{yr}^{-1}\right)\end{array}$ & $\begin{array}{r}V_{\mathrm{N}} \\
\left(\mathrm{mm} \mathrm{yr}^{-1}\right)\end{array}$ & $\begin{array}{r}V_{\mathrm{Up}} \\
\left(\mathrm{mm} \mathrm{yr}^{-1}\right)\end{array}$ & $\begin{array}{r}\sigma_{\mathrm{E}} \\
\left(\mathrm{mm} \mathrm{yr}^{-1}\right)\end{array}$ & $\begin{array}{r}\sigma_{\mathrm{N}} \\
\left(\mathrm{mm} \mathrm{yr}^{-1}\right)\end{array}$ & $\begin{array}{r}\sigma_{\mathrm{Up}} \\
\left(\mathrm{mm} \mathrm{yr}^{-1}\right)\end{array}$ & $\begin{array}{r}\text { Dur. } \\
(\mathrm{yr})\end{array}$ \\
\hline MORN & 0.2725 & 45.6939 & 231.1 & 0.71 & 0.19 & -0.26 & 0.07 & 0.06 & 0.14 & 5.89 \\
\hline MSGT & 1.6294 & 42.8796 & 548.6 & -0.52 & 0.43 & -0.52 & 0.08 & 0.04 & 0.08 & 6.10 \\
\hline MSMM & 6.1998 & 43.8107 & 797.8 & 0.45 & 0.54 & 0.90 & 0.17 & 0.04 & 1.59 & 4.10 \\
\hline MSRT & 1.5214 & 45.5414 & 566.2 & -0.40 & 0.30 & -0.49 & 0.13 & 0.07 & 0.69 & 3.86 \\
\hline MTDM & -0.4849 & 43.8827 & 133.9 & 0.64 & 0.19 & -0.94 & 0.10 & 0.06 & 0.24 & 6.32 \\
\hline MTPL* & 3.8648 & 43.6374 & 120.3 & 0.06 & -0.07 & 0.02 & 0.03 & 0.01 & 0.04 & 13.89 \\
\hline NICA* & 7.2273 & 43.7033 & 256.5 & -0.09 & 0.00 & 0.17 & 0.05 & 0.04 & 0.09 & 11.04 \\
\hline NICE* & 7.3003 & 43.7255 & 440.6 & 0.17 & 0.05 & 0.39 & 0.04 & 0.02 & 0.09 & 13.04 \\
\hline NIME* & 4.3569 & 43.8286 & 106.1 & -0.07 & 0.23 & -0.40 & 0.04 & 0.03 & 0.03 & 7.79 \\
\hline ORON & -1.6090 & 43.1390 & 204.9 & 0.36 & 0.26 & 0.12 & 0.05 & 0.10 & 0.13 & 5.36 \\
\hline PALI & 4.8105 & 43.3760 & 60.5 & -0.17 & 0.49 & -1.19 & 0.14 & 0.05 & 0.20 & 6.06 \\
\hline PAMP & -1.6360 & 42.8060 & 524.5 & -0.14 & 0.06 & -0.29 & 0.17 & 0.12 & 0.10 & 5.36 \\
\hline PERP & 2.8820 & 42.6891 & 95.9 & 1.32 & 0.50 & -0.04 & 1.30 & 0.24 & 0.13 & 6.78 \\
\hline PIAA & 8.6293 & 42.2350 & 543.3 & 0.27 & 0.79 & -2.40 & 1.06 & 0.08 & 1.24 & 3.18 \\
\hline PIAN & 9.0561 & 41.4947 & 153.1 & 1.21 & -0.53 & -0.46 & 0.21 & 0.20 & 0.52 & 3.63 \\
\hline PIMI & 0.1426 & 42.9364 & 2923.4 & 1.78 & -5.46 & 2.29 & 1.35 & 11.05 & 2.11 & 3.22 \\
\hline PLOE & -3.4273 & 47.7461 & 74.0 & 0.19 & 0.21 & -0.74 & 0.11 & 0.17 & 0.19 & 7.04 \\
\hline POBU & 4.1603 & 46.3828 & 420.8 & 0.48 & 0.17 & -1.92 & 0.14 & 0.03 & 0.39 & 5.97 \\
\hline PQRL* & 6.2061 & 42.9833 & 112.3 & 0.18 & 0.02 & -0.05 & 0.09 & 0.08 & 0.18 & 10.29 \\
\hline PRNY & 6.3383 & 46.9049 & 883.6 & -1.15 & 0.17 & -0.33 & 0.94 & 0.09 & 0.47 & 6.16 \\
\hline PUYA & 6.4789 & 44.8577 & 1690.3 & 0.47 & 0.23 & 1.24 & 0.15 & 0.08 & 0.10 & 8.08 \\
\hline PUYV* & 3.8789 & 45.0436 & 710.3 & -0.07 & -0.11 & -0.13 & 0.05 & 0.06 & 0.12 & 9.30 \\
\hline RABU* & 6.9771 & 44.2678 & 2551.9 & 0.33 & -0.10 & 0.30 & 0.32 & 0.10 & 0.25 & 8.66 \\
\hline RAYL & 6.4939 & 43.1605 & 336.3 & -0.17 & 0.87 & -2.07 & 0.24 & 0.06 & 0.32 & 5.21 \\
\hline RIOJ & -2.5000 & 42.4600 & 503.1 & 1.04 & -1.48 & -0.03 & 0.45 & 2.11 & 0.63 & 6.47 \\
\hline ROSD* & 6.6282 & 45.6915 & 1694.5 & -0.28 & -0.13 & 1.88 & 0.31 & 0.11 & 0.24 & 8.04 \\
\hline ROTG & -3.9657 & 48.7184 & 56.1 & -0.38 & 0.14 & -0.51 & 0.78 & 0.12 & 0.44 & 4.35 \\
\hline ROYA* & -1.0244 & 45.6386 & 69.0 & 0.38 & 0.06 & 0.04 & 0.07 & 0.05 & 0.15 & 6.78 \\
\hline RST2 & 5.4837 & 43.9410 & 1069.8 & 0.01 & 0.30 & 0.05 & 0.05 & 0.11 & 0.12 & 6.06 \\
\hline RSTL* & 5.4837 & 43.9410 & 1069.9 & -0.11 & 0.02 & -0.36 & 0.03 & 0.06 & 0.59 & 11.43 \\
\hline SABL & -1.8058 & 46.5300 & 56.7 & 0.02 & -0.55 & 0.40 & 0.16 & 0.10 & 0.15 & 9.91 \\
\hline SANG & -1.2870 & 42.5800 & 459.2 & 0.43 & -0.06 & 0.92 & 0.08 & 0.04 & 0.24 & 5.36 \\
\hline SARI & 9.4025 & 41.8583 & 57.2 & 0.04 & 0.55 & -0.98 & 0.15 & 0.12 & 0.15 & 3.63 \\
\hline SAUV* & 4.4675 & 44.2554 & 406.4 & -0.29 & -0.12 & -0.17 & 0.05 & 0.03 & 0.14 & 13.99 \\
\hline SBAR & 2.1740 & 41.9800 & 937.9 & -0.01 & -0.30 & -0.18 & 0.05 & 0.05 & 0.16 & 7.72 \\
\hline SCDA* & 3.2675 & 44.7945 & 1115.3 & -0.21 & 0.16 & -0.50 & 0.11 & 0.03 & 0.22 & 6.61 \\
\hline SCLP & 4.4264 & 45.7503 & 703.7 & 0.30 & -0.20 & -0.63 & 0.16 & 0.05 & 0.18 & 8.44 \\
\hline SCOA & -1.6800 & 43.4000 & 59.5 & 0.01 & 0.14 & -2.77 & 0.11 & 0.06 & 0.19 & 8.02 \\
\hline SEUR* & 5.1515 & 46.9943 & 245.1 & 0.19 & 0.05 & 0.10 & 0.08 & 0.01 & 0.15 & 7.28 \\
\hline SJDV* & 4.6766 & 45.8790 & 432.4 & 0.00 & -0.07 & -0.03 & 0.02 & 0.02 & 0.06 & 13.98 \\
\hline SJPL & 2.6896 & 45.6632 & 1029.1 & 0.99 & 0.38 & -0.05 & 0.25 & 0.19 & 0.36 & 5.21 \\
\hline SLVT & 3.2683 & 43.9198 & 811.8 & 0.19 & 0.27 & 0.25 & 0.17 & 0.05 & 0.16 & 6.29 \\
\hline SMLE & -0.2219 & 46.4112 & 170.5 & 0.69 & 0.27 & -0.13 & 0.11 & 0.11 & 0.10 & 6.63 \\
\hline SMTG & -2.0275 & 48.6411 & 57.7 & 0.67 & 0.41 & -0.40 & 0.35 & 0.15 & 1.13 & 3.87 \\
\hline $\mathrm{SOPH}^{*}$ & 7.0544 & 43.6114 & 193.3 & 0.04 & -0.05 & 0.21 & 0.04 & 0.02 & 0.07 & 12.96 \\
\hline SORI & 1.1330 & 42.3750 & 1284.5 & 0.33 & -0.12 & -1.15 & 0.22 & 0.09 & 0.22 & 7.89 \\
\hline SOUS & 2.0271 & 44.8746 & 597.7 & 0.04 & 0.20 & 0.11 & 0.18 & 0.04 & 0.56 & 6.63 \\
\hline STEY* & 5.7618 & 45.2352 & 1394.9 & -0.03 & -0.02 & 2.16 & 0.14 & 0.04 & 0.68 & 10.46 \\
\hline STJ9* & 7.6838 & 48.6217 & 237.2 & -0.03 & 0.01 & 0.23 & 0.02 & 0.03 & 0.14 & 13.98 \\
\hline STMR & 4.4216 & 43.4491 & 56.2 & 0.10 & 0.04 & -2.23 & 0.11 & 0.17 & 0.29 & 5.72 \\
\hline STPS* & 3.2940 & 46.3084 & 299.7 & -0.24 & 0.09 & 0.07 & 0.10 & 0.03 & 0.21 & 7.28 \\
\hline STV2 & 6.1062 & 44.5669 & 814.7 & -0.68 & 0.17 & 1.13 & 0.25 & 0.17 & 0.55 & 5.04 \\
\hline TAFA & -1.6770 & 42.5210 & 473.9 & 0.12 & -0.18 & -0.20 & 0.05 & 0.08 & 0.22 & 5.36 \\
\hline TENC* & 4.2875 & 45.1246 & 936.5 & 0.20 & 0.06 & 0.05 & 0.03 & 0.03 & 0.09 & 9.53 \\
\hline TLMF* & 1.3751 & 43.5746 & 221.1 & -0.11 & 0.22 & -0.80 & 0.09 & 0.02 & 0.35 & 11.53 \\
\hline TLSE* & 1.4800 & 43.5600 & 207.2 & 0.10 & -0.20 & 0.39 & 0.03 & 0.03 & 0.11 & 12.98 \\
\hline TORI* & 7.6613 & 45.0634 & 310.4 & 0.00 & 0.00 & 0.90 & 0.11 & 0.02 & 0.10 & 13.98 \\
\hline
\end{tabular}


Table 3. Continued.

\begin{tabular}{|c|c|c|c|c|c|c|c|c|c|c|}
\hline $\begin{array}{l}\text { Site } \\
\text { name }\end{array}$ & $\begin{array}{l}\text { Long. } \\
\left({ }^{\circ} \mathrm{E}\right)\end{array}$ & $\begin{array}{l}\text { Lat. } \\
\left({ }^{\circ} \mathrm{N}\right)\end{array}$ & $\begin{array}{l}\text { Alt. } \\
\text { (m) }\end{array}$ & $\begin{array}{r}V_{\mathrm{E}} \\
\left(\mathrm{mm} \mathrm{yr}^{-1}\right)\end{array}$ & $\begin{array}{r}V_{\mathrm{N}} \\
\left(\mathrm{mm} \mathrm{yr}^{-1}\right)\end{array}$ & $\begin{array}{r}V_{\mathrm{Up}} \\
\left(\mathrm{mm} \mathrm{yr}^{-1}\right)\end{array}$ & $\begin{array}{r}\sigma_{\mathrm{E}} \\
\left(\mathrm{mm} \mathrm{yr}^{-1}\right)\end{array}$ & $\begin{array}{r}\sigma_{\mathrm{N}} \\
\left(\mathrm{mm} \mathrm{yr}^{-1}\right)\end{array}$ & $\begin{array}{r}\sigma_{\mathrm{Up}} \\
\left(\mathrm{mm} \mathrm{yr}^{-1}\right)\end{array}$ & $\begin{array}{r}\text { Dur. } \\
(\mathrm{yr})\end{array}$ \\
\hline TOUL & 1.4810 & 43.5610 & 207.1 & -5.75 & 4.64 & -0.41 & 1.95 & 1.76 & 1.15 & 0.99 \\
\hline TREM* & -0.7940 & 47.1159 & 166.5 & 0.29 & -0.17 & 0.05 & 0.09 & 0.05 & 0.15 & 6.39 \\
\hline TRMO & 2.7248 & 44.2854 & 810.9 & 0.62 & 0.09 & -0.91 & 0.20 & 0.08 & 0.30 & 5.42 \\
\hline TROP* & 6.6010 & 43.2195 & 369.3 & -0.18 & 0.22 & -0.22 & 0.06 & 0.03 & 0.07 & 8.31 \\
\hline TUDE & -1.6030 & 42.0490 & 342.0 & 0.02 & -0.44 & -0.23 & 0.12 & 0.07 & 0.40 & 5.36 \\
\hline UNME & -0.3257 & 43.3216 & 278.7 & 0.09 & 0.56 & 0.34 & 0.10 & 0.05 & 0.25 & 6.54 \\
\hline VAUD & 5.6269 & 46.9812 & 271.5 & 0.79 & 0.41 & -0.37 & 0.28 & 0.10 & 0.41 & 5.86 \\
\hline VFCH & 1.7197 & 47.2942 & 153.3 & 0.32 & -0.53 & 0.39 & 0.05 & 0.03 & 0.11 & 12.31 \\
\hline VILR & 5.5518 & 45.0725 & 1076.7 & -0.17 & 0.32 & -0.82 & 0.13 & 0.05 & 0.13 & 7.06 \\
\hline VISN & 4.9492 & 44.3199 & 245.8 & -0.02 & 0.30 & -0.51 & 0.15 & 0.02 & 0.20 & 6.39 \\
\hline VOUR* & 4.7807 & 45.6616 & 324.9 & 0.42 & 0.10 & -2.77 & 0.54 & 0.32 & 0.90 & 7.66 \\
\hline VSOL & 6.0666 & 47.6894 & 321.9 & 0.27 & -0.20 & -0.82 & 0.12 & 0.23 & 0.25 & 4.71 \\
\hline WLBH & 7.3513 & 48.4152 & 819.0 & -0.43 & 0.07 & -2.61 & 0.09 & 0.04 & 0.36 & 13.18 \\
\hline ZARA* & -0.8800 & 41.6300 & 296.1 & -0.18 & -0.18 & -0.31 & 0.06 & 0.03 & 0.23 & 7.58 \\
\hline
\end{tabular}

Figure 7 shows the dispersion of the east and north components of the residual velocities. The 65 sites with time series longer than 8 years, horizontal velocities are lower than $0.7 \mathrm{~mm} \mathrm{yr}^{-1}$ (except CLAP) and the weighted rootmean-square (WRMS) dispersion is $0.18 \mathrm{~mm} \mathrm{yr}^{-1}$. For stations with longer time series of more than 10 or 12 years, the WRMS are similar $\left(0.17\right.$ and $0.18 \mathrm{~mm} \mathrm{yr}^{-1}$, respectively). This stability of the dispersion for stations over 8 years raises the question of whether these velocities represent a long-term motion of the sites or if we are reaching the threshold of the GPS horizontal velocity accuracy.

Deformation patterns associated with our velocity field are estimated by running an interpolation and smoothing function through the 3-D velocity data. Horizontal strain rates are calculated on a regular $0.5 \times 0.5^{\circ}$ grid using a Gaussian interpolation function with a $75 \mathrm{~km}$ half-width and taking into account each GPS sites uncertainty (cf., Mazzotti et al., 2011). The horizontal strain rate field (Fig. 8) shows no significant strain rates above $\sim 1 \times 10^{-9} \mathrm{yr}^{-1}$, except in the western Pyrenees where north-south to northeast-southwest extension is observed up to $\sim 4 \times 10^{-9} \mathrm{yr}^{-1}$, above the formal uncertainty level. This strain rate pattern is consistent with that derived from shorter time series of the Spanish continuous sites (Asensio et al., 2012) and campaign GPS data in the Pyrenees, where Rigo et al. (2015) estimate a north-south extension of $\sim 2 \times 10^{-9} \mathrm{yr}^{-1}$ in the western region but no significant strain in the central and eastern regions. In contrast, our estimation of non-significant strain rates $\left(<1 \times 10^{-9} \mathrm{yr}^{-1}\right)$ in the Western Alps does not agree with campaign GPS results in the Briançon region where east-west extension rates of $(16 \pm 11) \times 10^{-9} \mathrm{yr}^{-1}$ are estimated (Walpersdorf et al., 2015). This disagreement may be due to the high spatial wavelength of our solution ( $\sim 100 \mathrm{~km}$ or more), whereas campaign data from Walpersdorf et al. (2015) sample a much smaller area $(\sim 30 \mathrm{~km})$, and to the fact that we have less sites in Italy in our solution.
The main feature of the vertical velocities (Fig. 9) is the zone of uplift (up to $\sim 2.0 \mathrm{~mm} \mathrm{yr}^{-1}$ ) observed in the northcentral region of the Western Alps. This pattern of uplift is clearly limited to the region north of about $44.5^{\circ} \mathrm{N}$, whereas the southern French Alps and the foreland regions of the Rhone Valley and the French Jura show no vertical motion within $\pm 0.5 \mathrm{~mm} \mathrm{yr}^{-1}$. Our results are consistent with the uplift pattern identified by Serpelloni et al. (2013) in the Western Alps (average difference at common sites of $0.05 \pm 0.79 \mathrm{~mm} \mathrm{yr}^{-1}$ ), although the higher station density in our solution allows a sharper definition of region affected by significant uplift. In contrast, no significant vertical pattern can be observed in the Pyrenees, where most stations show vertical velocities about $\pm 0.5 \mathrm{~mm} \mathrm{yr}^{-1}$.

Stations in the northern region of the Western Alps show a first-order correlation between uplift rates and topography (Fig. 10), with highest uplift rates $\left(>1.0 \mathrm{~mm} \mathrm{yr}^{-1}\right)$ limited to stations located above $\sim 1000 \mathrm{~m}$ altitude. This is consistent with GPS and leveling results in the Swiss Alps where the most elevated region displays uplift rates up to $2 \mathrm{~mm} \mathrm{yr}^{-1}$ (Brockmann et al., 2012). Although this correlation seems straightforward, it is not the altitude of the GPS site that matters but rather the mean elevation of the mountains surrounding the site. In other words a GPS site in a valley located in the highest part of the western Alps should have a high uplift rate even if it is located at low elevation. Because GPS stations are commonly located on ridges rather than in valleys, station elevations are a good proxy for the mean elevation of the surrounding area. To check this later assumption, we have filtered the topography by averaging it over $10 \times 10 \mathrm{~km}$ windows and the correlation is consistent with the results presented in Fig. 10. In the Western Alps, the southern part shows no evidences of significant uplift $\left(\sim 0 \mathrm{~mm} \mathrm{yr}^{-1}\right)$, in contrast to the northern part of the Western Alps, where the uplift rates are the highest $\left(1-2 \mathrm{~mm} \mathrm{yr}^{-1}\right)$ for the same elevation range. To first order these uplift rates seems correlated to 

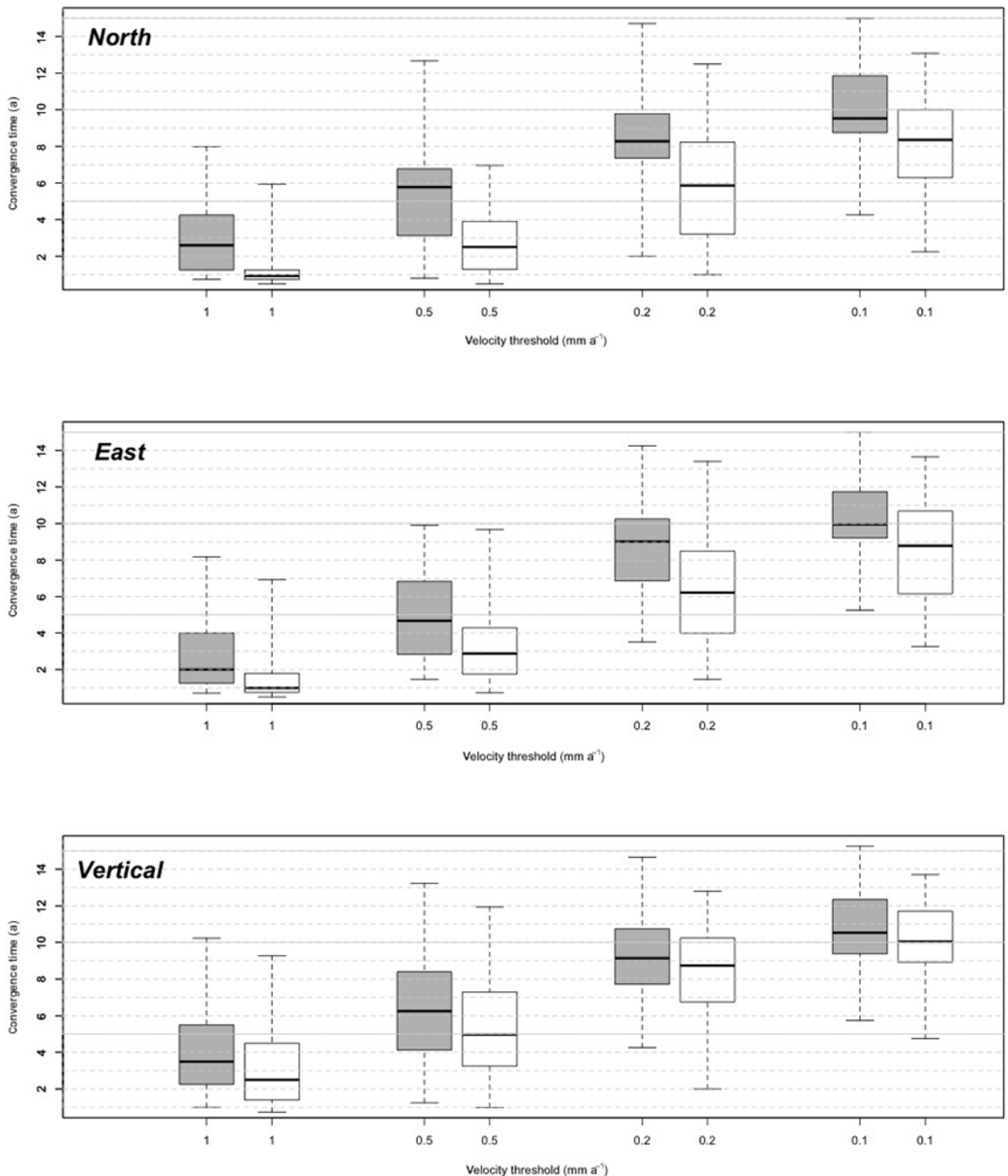

Figure 4. Whisker plots of convergence time for four velocity thresholds $\left(1.0,0.5,0.2\right.$, and $\left.0.1 \mathrm{~mm} \mathrm{yr}^{-1}\right)$. Thick bars, boxes, and dashed bars represent the median, quartile $(25$ and $75 \%)$ and extreme values of the distributions for the 44 stations with at least 10 years of data. Grey and white whisker plots correspond to unfiltered (raw) and filtered (with common-mode correction) data.

the Würmian ice cap that was thicker in the northern part of the Western Alps (Stocchi et al., 2005) and can be modeled according to Chéry et al. (2016).

Stations in the Pyrenees show no such correlation, in particular for the sites with the longest time series and above an elevation of $1500 \mathrm{~m}$, which show vertical velocities of $\sim 0.1 \pm 0.2 \mathrm{~mm} \mathrm{yr}^{-1}$ (Figs. 9 and 10). This very low uplift rate is in agreement with an erosion-induced deformation in the Pyrenees (Genti et al., 2015). Figure 10 shows no correlation between vertical velocities and time-series lengths for the Western Alps or the Pyrenees, but it clearly shows the Pyrenees's lack of continuous GPS sites at mid- to high elevations. 

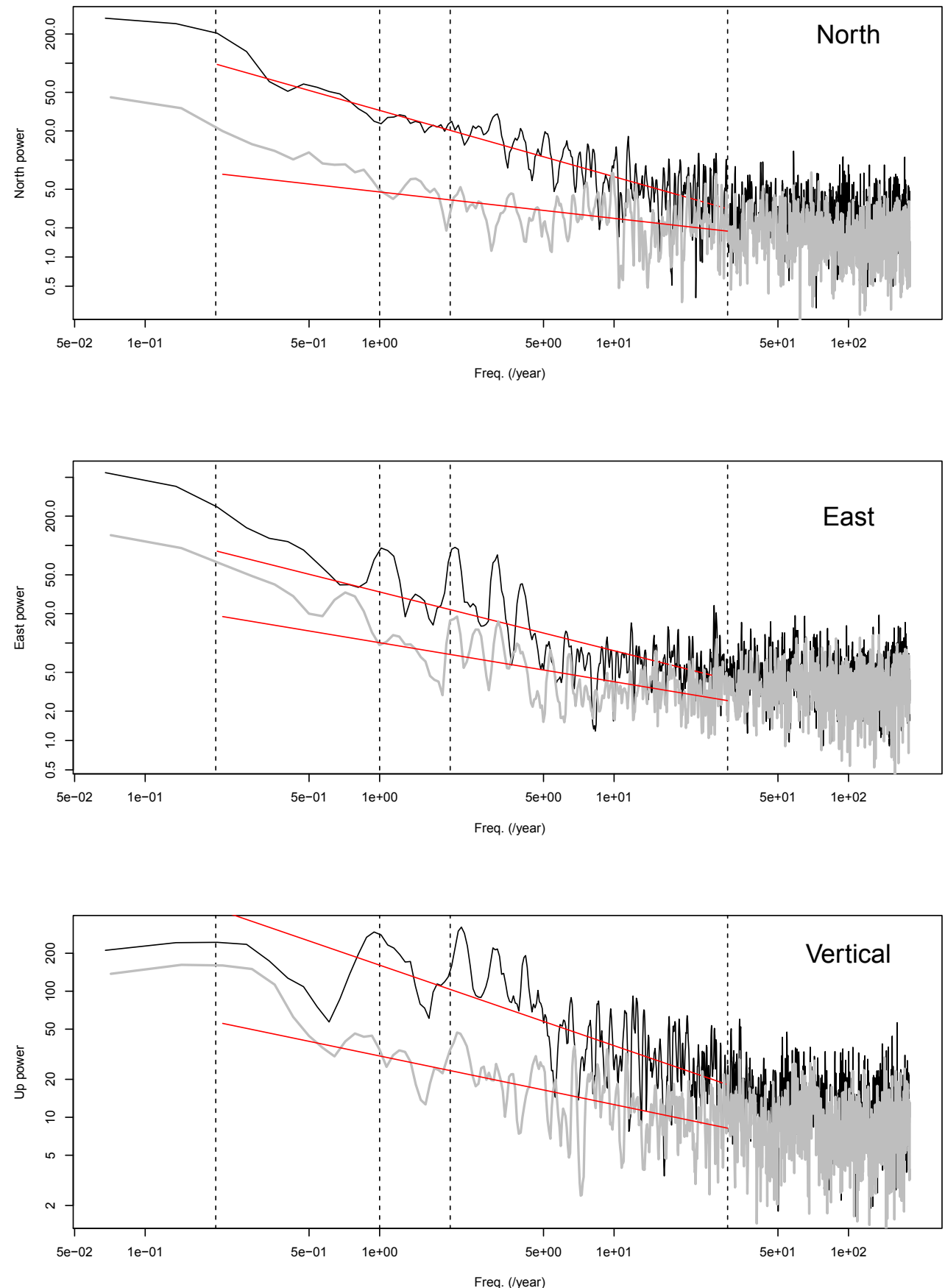

Figure 5. Noise spectra for site MTPL. Spectra derived form the unfiltered time series are in black, those for the filtered time series are in grey. The red lines represent the best spectral models used for the uncertainty analyses.

The dispersion of the horizontal velocities (Fig. 7) and vertical velocity vs. altitude correlation (Fig. 10) shows that we can resolve sub-millimeter horizontal and vertical velocities in the Alps and the Pyrenees. Furthermore, the independent result obtained with leveling in the Western Alps (Nocquet et al., 2016) shows that the uplift motion on the order of $1 \mathrm{~mm} \mathrm{yr}^{-1}$ is significant. Strain rate computation (Fig. 8) suggests consistent patterns such as $\mathrm{E}-\mathrm{W}$ extension in the Western Alps. However, these rates $\left(\sim 1 \times 10^{-9} \mathrm{yr}^{-1}\right)$ are not significant and only denser networks with careful analysis of local coherent patterns will tell us whether this pattern is true or not. Combining the continuous and survey GNSS networks in a single homogeneous reprocessing could achieve this. 


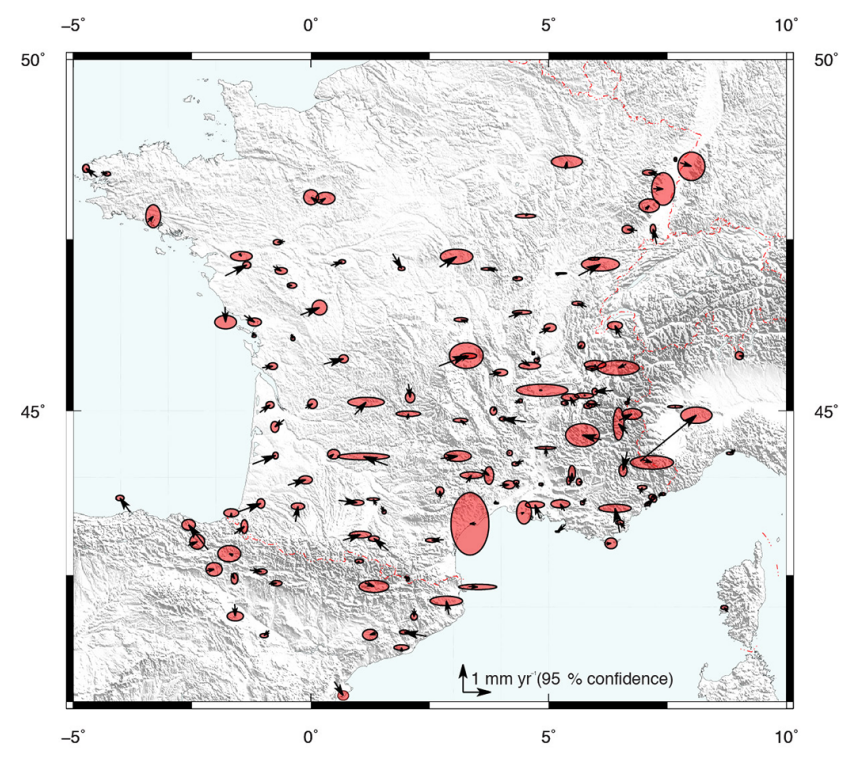

Figure 6. Horizontal velocities for stations with time series longer than 5 years in a regional (western Europe) reference frame.

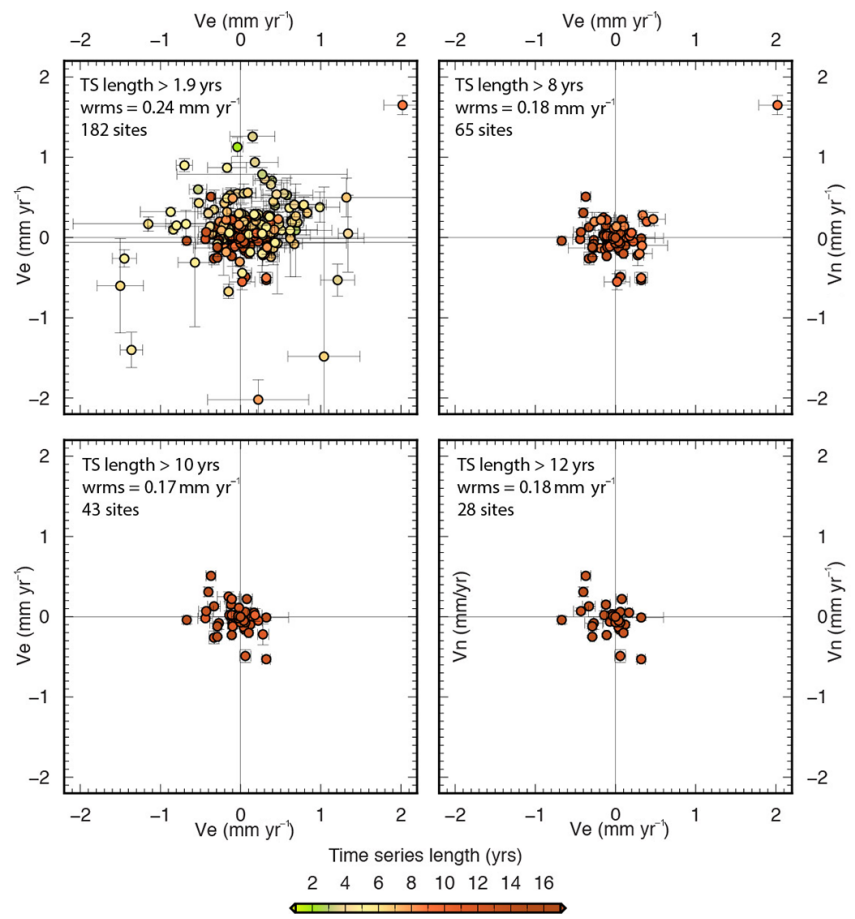

Figure 7. East vs. north components of the residuals relative to a regional reference, color-coded based on the time-series lengths.

To the question "Are the Alps collapsing?" (Selverstone, 2005), we can answer "not in the sense of a post-orogenic collapse of the mountain range". Indeed, our GPS velocities show no significant $\left(<0.2 \mathrm{~mm} \mathrm{yr}^{-1}\right)$ horizontal outward flowing of the Western Alps. However, significant uplift of the northern part of the Western Alps is clearly observed.

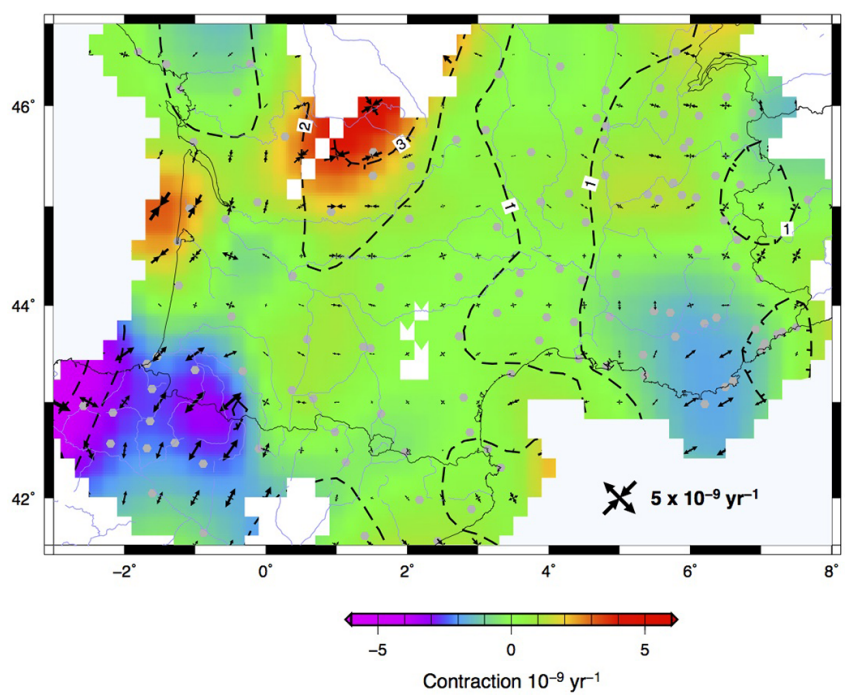

Figure 8. Smoothed horizontal strain rate field for the Western Alps and Pyrenees region. Dashed lines are strain rate standard error. Light grey curves show the drainage pattern and dark grey curves the seashores and country borders.

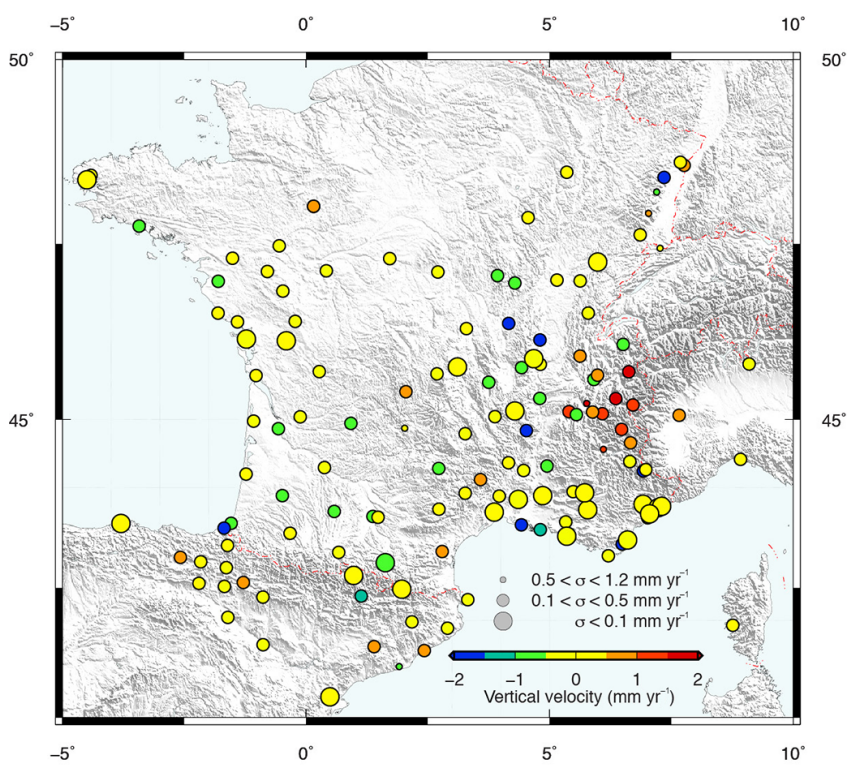

Figure 9. Vertical velocities for stations with time series longer than 5 years in the IGS08 reference frame (see Table 3 for the site list).

These vertical velocities seem correlated to the Würmian ice cap thickness and suggest that part of the present-day uplift is related to postglacial rebound. Models of glacial isotactic rebound for the Alps suggest either a low amplitude and a large wavelength (Stocchi et al., 2005) or a very localized uplift due to the Little Ice Age glacier shrinkage (Barletta et al., 2006); both models are not consistent with the GPS observations that suggest a short wavelength and a large amplitude of the uplift. The drawback of these models is that 

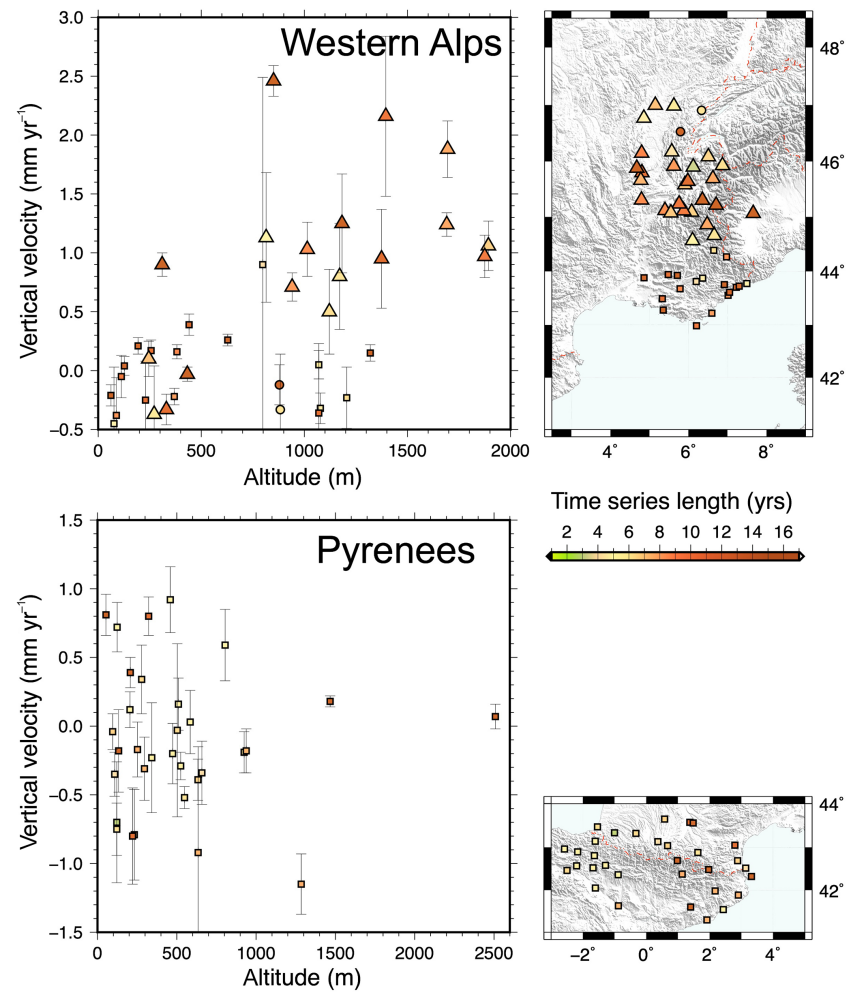

Time series length (yrs) \begin{tabular}{llllllll}
2 & 4 & 6 & 8 & 10 & 12 & 14 & 16 \\
\hline
\end{tabular}
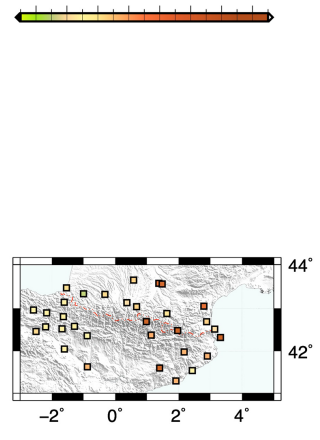

Figure 10. Vertical velocities, color-coded based on the time-series length, vs. site elevations for stations located in the Western Alps and the Pyrenees.

they consider a 1-D-layered lithosphere, which is probably far from the truth given the geological history of the region. An evidence for a more complicated lithosphere is given by the seismicity below Switzerland where the earthquake maximum depth vary from $\sim 30 \mathrm{~km}$ in the foreland to $\sim 15 \mathrm{~km}$ below the range (Deichmann, 2003). This suggests a wedge of softer material in the lower crust below the Alps (Chéry et al., 2016), in some way similar to the Andes of Patagonia where a localized uplift due to glacier mass changes is modeled based on a wedge of softer material in the continental lithosphere (Klemann et al., 2007). This does not exclude contributions from deep processes for part of the uplift (Fox et al., 2015) or erosion-induced rebound (Champagnac et al., 2007; Genti et al., 2015; Vernant et al., 2013). A careful and detailed model of the response to the Last Glacial Maximum of the Pyrenees and the Alps will certainly bring valuable new insights on post-orogenic mountain processes.

\section{Conclusions}

Our new GPS velocity solution based on 182 sites for the region encompassing the Western Alps, the Pyrenees, and southern France leads to four main conclusions:
1. These areas can be associated with as a stable region, within a dispersion (WRMS) of $\sim 0.2 \mathrm{~mm} \mathrm{yr}^{-1}$ on the horizontal velocities. However, even with this low dispersion, horizontal velocities, without a regional coherence, can still reach up to $0.7 \mathrm{~mm} \mathrm{yr}^{-1}$, raising the question of the significance of these velocities: are there local processes explaining these residuals or do they represent a bias in our uncertainty estimates?

2. Significant horizontal strain rates up to $4 \times 10^{-9} \mathrm{yr}^{-1}$ of NNE-SSW extension are evidenced in the Western Pyrenees, but none are seen in the Western Alps (i.e., $\left.<1 \times 10^{-9} \mathrm{yr}^{-1}\right)$.

3. In contrast, significant uplift rates up to $2 \mathrm{~mm} \mathrm{yr}^{-1}$ are observed in the Western Alps but not in the Pyrenees where they are close to $0.1 \pm 0.2 \mathrm{~mm} \mathrm{yr}^{-1}$.

4. These significant uplift rates are observed in the northern part of the Western Alps. They are correlated to the mean elevation of the region surrounding the GPS sites, but this correlation is not true for the Pyrenees or the southern part of the Western Alps.

The observations of uplift rates, and lack of significant horizontal motion in the northern Western Alps, are correlated with the Würmian ice cap thickness and tend to suggest that a large part of this uplift is induced by postglacial rebound from the Last Glacial Maximum associated with a wedge of softer material in the continental lithosphere below the Western Alps. Unfortunately, the GPS network is too sparse to characterize uplift in the Pyrenees, where we see significant horizontal strain rates. With a few more sites in this region we will be able, with a careful comparison between the Pyrenees, the southern and northern regions of the Western Alps, to decipher the role of erosion, glacier mass changes, and deep processes in the present-day uplift rates of this mountain ranges.

\section{Data availability}

We thank Natural Resources Canada for providing the CCRS-PPP software. Time-series analysis was done with the R statistical software (http://www.r-project.org). GPS data used in this study were extracted from the following archives: RENAG (http://renag.resif.fr), IGS (http://www.igs.org), EUREF (http://www.epncb.oma.be), RGP (http://rgp.ign.fr), Topo-Iberia (http://www.igme.es/TopoIberia/default.html), RGAN (http://www.navarra.es/appsext/rgan/default.aspx), EPSH (http://epsh.unizar.es/ serreta/huescagps.html), and CATNET (http://catnet-ip.icc.cat).

Acknowledgements. We thank E. Brockmann and two anonymous reviewers for their helpful reviews. This work was supported by the Centre National de la Recherche Scientifique (CNRS-INSU), 
Languedoc-Roussillon Regional Authority (grant Chercheurs d'Avenir to P. Vernant), and the REseau SIsmologique \& géodésique Français (RESIF). We thank all the people involved in the GPS site maintenance and data distribution for the stations used in this study.

Edited by: S. McClusky

Reviewed by: E. Brockmann and two anonymous referees

\section{References}

Agnew, D. C.: The time-domain behaviour of power-law noises, Geophys. Res. Lett., 19, 333-336, 1992.

Alasset, P.-J. and Meghraoui, M.: Active faulting in the western Pyrénées (France): Paleoseismic evidence for late Holocene ruptures, Tectonophysics, 409, 39-54, doi:10.1016/j.tecto.2005.08.019, 2005.

Altamimi, Z., Collilieux, X., and Métivier, L.: ITRF2008: an improved solution of the international terrestrial reference frame, J. Geodesy., 85, 457-473, doi:10.1007/s00190-011-0444-4, 2011.

Argus, D. F., Gordon, R. G., Heflin, M. B., Ma, C., Eanes, R. J., Willis, P., Peltier, W. R., and Owen, S. E.: The angular velocities of the plates and the velocity of Earth's centre from space geodesy, Geophys. J. Int., 180, 913-960, doi:10.1111/j.1365246X.2009.04463.x, 2010.

Argus, D. F., Peltier, W. R., and Watkins, M. M.: Glacial isostatic adjustment observed using very long baseline interferometry and satellite laser ranging geodesy, J. Geophys. Res., 104, 2907729093, 1999.

Asensio, E., Khazaradze, G., Echeverria, A., King, R. W., and Vilajosana, I.: GPS studies of active deformation in the Pyrenees, Geophys. J. Int., 190, 913-921, doi:10.1111/j.1365246X.2012.05525.x, 2012.

Barletta, V. R., Ferrari, C., Diolaiuti, G., Carnielli, T., Sabadini, R., and Smiraglia, C.: Glacier shrinkage and modeled uplift of the Alps, Geophys. Res. Lett., 33, L14307, doi:10.1029/2006GL026490, 2006.

Blewitt, G. and Lavallée, D.: Effect of annual signals on geodetic velocity, J. Geophys. Res., 107, ETG_9-1-ETG_9-11, 2002.

Boehm, J., Werl, B., and Schuh, H.: Troposphere mapping functions for GPS and very long baseline interferometry from European Centre for Medium-Range Weather Forecasts operational analysis data, J. Geophys. Res.-Sol. Ea., 111, B02406, doi:10.1029/2005JB003629, 2006.

Brockmann, E., Ineichen, D., Marti, U., Schaer, S., and Schlatter, A.: Determination of Tectonic Movements in the Swiss Alps using GNSS and Levelling, in: Geodesy for planet Earth: International Association of Geodesy Symposia, edited by: Kenyon, S., Pacino, M. C., and Marti, U., 136, 689-695, 2012.

Champagnac, J. D., Molnar, P., Anderson, R. S., Sue, C., and Delacou, B.: Quaternary erosion-induced isostatic rebound in the western Alps, Geology, 35, 195-198, doi:10.1130/g23053a.1, 2007.

Chardon, D., Hermitte, D., Nguyen, F., and Bellier, O.: First paleoseismological constraints on the strongest earthquake in France (Provence) in the twentieth century, Geology, 33, 901-904, doi:10.1130/G21713.1, 2005.
Chéry, J., Genti, M., and Vernant, P.: Ice cap melting and low-viscosity crustal root explain the narrow geodetic uplift of the Western Alps, Geophys. Res. Lett., 43, 1-8, doi:10.1002/2016GL067821, 2016.

Chevrot, S., Sylvander, M., and Delouis, B.: A preliminary catalog of moment tensors for the Pyrenees, Tectonophysics, 510, 239251, doi:10.1016/j.tecto.2011.07.011, 2011.

Collilieux, X., Altamimi, Z., Métivier, L., and van Dam, T.: Quality assessment of GPS reprocessed Terrestrial Reference Frame, Amer. Geophys. Union Fall Meeting, San Francisco, 2009.

Deichmann, N.: Focal depths of earthquakes below Switzerland, Swiss Seismol Service ETH Zürich, 2003.

Fox, M., Herman, F., Kissling, E., and Willett, S. D.: Rapid exhumation in the Western Alps driven by slab detachment and glacial erosion, Geology, 43, 379-382, doi:10.1130/G36411.1, 2015.

Genti, M., Chery, J., Vernant, P., and Rigo, A.: Impact of gravity forces and topography denudation on normal faulting in CentralWestern Pyrenees: Insights from 2-D numerical models, C. R. Geosci., 348, 1-11, doi:10.1016/j.crte.2015.08.004, 2015.

Héroux, P. and Kouba, J.: GPS Precise Point Positioning using IGS orbit pro- ducts, Phys. Chem. Earth, 26, 573-578, 2001.

Kierulf, H. P., Ouassou, M., Simpson, M. J. R., and Vestøl, O.: A continuous velocity field for Norway, J. Geodesy., 87, 337-349, doi:10.1007/s00190-012-0603-2, 2013.

Klemann, V., Ivins, E. R., Martinec, Z., and Wolf, D.: Models of active glacial isostasy roofing warm subduction: Case of the South Patagonian Ice Field, J. Geophys. Res.-Sol. Ea., 112, B09405, doi:10.1029/2006JB004818, 2007.

Kouba, J.: A guide to using International GNSS Service (IGS) products, International GNSS Service, available at: https://igscb. jpl.nasa.gov/components/usage.html (last access: 20 February 2016), 2009.

Lacan, P. and Ortuño, M.: Active Tectonics of the Pyrenees: A review, J. Iber. Geol., 38, 9-30, 2012.

Larroque, C., Delouis, B., Godel, B., and Nocquet, J.-M.: Active deformation at the southwestern Alps-Ligurian basin junction (France-Italy boundary): Evidence for recent change from compression to extension in the Argentera massif, Tectonophysics, 467, 22-34, doi:10.1016/j.tecto.2008.12.013, 2009.

Lyard, F., Lefevre, F., Letellier, T., and Francis, O.: Modelling the global ocean tides: modern insights from FES2004, Ocean Dynam., 56, 394-415, doi:10.1007/s10236-006-0086-x, 2006.

Mazzotti, S., Leonard, L. J., Cassidy, J. F., Rogers, G. C., and Halchuk, S.: Seismic hazard in western Canada from GPS strain rates versus earthquake catalog: J. Geophys. Res.-Sol.Ea., 116, B12310, doi:10.1029/2011JB008213, 2011.

Nocquet, J. M. and Calais, E.: Crustal velocity field of the western Europe from permanent GPS array solutions, 1996-2001, Geophys. J. Int., 154, 72-88, 2003.

Nocquet, J.-M.: Present-day kinematics of the Mediterranean: A comprehensive overview of GPS results, Tectonophysics, 579, 220-242, doi:10.1016/j.tecto.2012.03.037, 2012.

Nocquet, J. M., Sue, C., Walpersdorf, A., Tran, T., Lenôtre, N., Vernant, P., Cushing, M., Jouanne, F., Masson, F., Baize, B., Chéry, J., and van der Beek, P. A.: Present-day uplift of the western Alps, Sci. Rep., 6, 1-6, doi:10.1038/srep28404, 2016.

Rebischung, P., Schmid, R., and Ray, J.: Upcoming switch to IGS08/igs08, atx. IGSMAIL-6354, 2011. 
Rigo, A., Vernant, P., Feigl, K. L., Goula, X., Khazaradze, G., Talaya, J., Morel, L., Nicolas, J., Baize, S., Chéry, J., and Sylvander, M.: Present-day deformation of the Pyrenees revealed by GPS surveying and earthquake focal mechanisms until 2011, Geophys. J. Int., 201, 947-964, doi:10.1093/gji/ggv052, 2015.

Rothacher, M. and Schmid, R.: ANTEX: The antenna exchange format version 1.3. Format specifications, IGS Central Bureau, Pasadena, 2006.

Santamaría-Gómez, A., Bouin, M.-N., Collilieux, X., and Wöppelmann, G.: Correlated errors in GPS position time series: Implications for velocity estimates, J. Geophys. Res.-Sol. Ea., 116, B01405, doi:10.1029/2010JB007701, 2011.

Selverstone, J.: Are the Alps collapsing?, Annu. Rev. Earth Planet. Sci., 33, 113-132, doi:10.1146/annurev.earth.33.092203.122535, 2005.

Serpelloni, E., Faccenna, C., Spada, G., Dong, D., and Williams, S. D.: Vertical GPS ground motion rates in the Euro-Mediterranean region: New evidence of velocity gradients at different spatial scales along the Nubia-Eurasia plate boundary, J. Geophys. Res.Sol. Ea., 118, 6003-6024, doi:10.1002/2013JB010102, 2013.

Stocchi, P., Spada, G., and Cianetti, S.: Isostatic rebound following the Alpine deglaciation: Impact on the sea level variations and vertical movements in the Mediterranean region, Geophys. J. Int., 162, 137-147, doi:10.1111/j.1365-246X.2005.02653.x, 2005.

Tregoning, P. and Watson, C.: Atmospheric effects and spurious signals in GPS analyses, J. Geophys. Res.-Sol. Ea., 114, B09403, doi:10.1029/2009JB006344, 2009.
Vernant, P., Hivert, F., Chéry, J., Steer, P., Cattin, R., and Rigo, A.: Erosion-induced isostatic rebound triggers extension in low convergent mountain ranges, Geology, 41, 467-470, doi:10.1130/G33942.1, 2013.

Vigny, C., Chéry, J., Duquesnoy, T., Jouanne, F., Ammann, J., Anzidei, M., Avouac, J. P., Barlier, F., Bayer, R., Briole, P., Calais, E., Cotton, F., Duquenne, F., Feigl, K. L., Ferhat, G., Flouzat, M., Gamond, J. F., Geiger, A., Harmel, A., Kasser, M., Laplanche, M., Le Pape, M., Martinod, J., Mnard, G., Meyer, B., Ruegg, J. C., Scheubel, J. M., Scotti, O. and Vidal, G.: GPS network monitors the Western Alps' deformation over a five-year period: 1993-1998, J. Geodesy., 76, 63-76, doi:10.1007/s00190001-0231-8, 2002.

Walpersdorf, A., Sue, C., Baize, S., Cotte, N., Bascou, P., Beauval, C., Collard, P., Daniel, G., Dyer, H., Grasso, J. R., Hautecoeur, O., Helmstetter, A., Hok, S., Langlais, M., Ménard, G., Mousavi, Z., Ponton, F., Rizza, M., Rolland, L., Souami, D., Thirard, L., Vaudey, P., Voisin, C. and Martinod, J.: Coherence between geodetic and seismic deformation in a context of slow tectonic activity (SW Alps, France), J. Geodynam., 85, 58-65, doi:10.1016/j.jog.2015.02.001, 2015.

Williams, S. D. P.: The effect of coloured noise on the uncertainties of rates estimated from geodetic time series, J. Geodesy, 76, 483494, doi:10.1007/s00190-002-0283-4, 2003. 